\title{
Anomalies, conformal manifolds, and spheres
}

\author{
Jaume Gomis, ${ }^{a}$ Po-Shen Hsin, ${ }^{b}$ Zohar Komargodski, ${ }^{c}$ Adam Schwimmer, ${ }^{c}$ \\ Nathan Seiberg ${ }^{d}$ and Stefan Theisen ${ }^{e}$ \\ ${ }^{a}$ Perimeter Institute for Theoretical Physics, \\ Waterloo, Ontario, N2L 2Y5, Canada \\ ${ }^{b}$ Department of Physics, Princeton University, \\ Princeton, NJ 08544, U.S.A. \\ ${ }^{c}$ Weizmann Institute of Science, \\ Rehovot 76100, Israel \\ ${ }^{d}$ School of Natural Sciences, Institute for Advanced Study, \\ Princeton, NJ 08540, U.S.A. \\ ${ }^{e}$ Max-Planck-Institut für Gravitationsphysik, Albert-Einstein-Institut, \\ 14476 Golm, Germany \\ E-mail: jgomis@perimeterinstitute.ca, nazgoulz@gmail.com, \\ zohar.komargodski@weizmann.ac.il, adam.schwimmer@weizmann.ac.il, \\ seiberg@ias.edu, stefan.theisen@aei.mpg.de
}

ABSTRACT: The two-point function of exactly marginal operators leads to a universal contribution to the trace anomaly in even dimensions. We study aspects of this trace anomaly, emphasizing its interpretation as a sigma model, whose target space $\mathcal{M}$ is the space of conformal field theories (a.k.a. the conformal manifold). When the underlying quantum field theory is supersymmetric, this sigma model has to be appropriately supersymmetrized. As examples, we consider in some detail $\mathcal{N}=(2,2)$ and $\mathcal{N}=(0,2)$ supersymmetric theories in $d=2$ and $\mathcal{N}=2$ supersymmetric theories in $d=4$. This reasoning leads to new information about the conformal manifolds of these theories, for example, we show that the manifold is Kähler-Hodge and we further argue that it has vanishing Kähler class. For $\mathcal{N}=(2,2)$ theories in $d=2$ and $\mathcal{N}=2$ theories in $d=4$ we also show that the relation between the sphere partition function and the Kähler potential of $\mathcal{M}$ follows immediately from the appropriate sigma models that we construct. Along the way we find several examples of potential trace anomalies that obey the Wess-Zumino consistency conditions, but can be ruled out by a more detailed analysis.

KEYWORDS: Supersymmetric gauge theory, Anomalies in Field and String Theories

ArXiv EPrint: 1509.08511 


\section{Contents}

1 Introduction 1

2 The anomaly associated with the metric on $\mathcal{M} \quad 3$

$3 \mathcal{N}=(2,2)$ supersymmetry in $d=2 \quad 7$

$4(0,2)$ supersymmetric theories $\quad 14$

$\begin{array}{lll}5 \mathcal{N}=2 \text { supersymmetry in } d=4 & 17\end{array}$

$\begin{array}{ll}\text { A Normalization of the anomaly } & 21\end{array}$

$\begin{array}{ll}\text { B The FTPR operator and its properties } & 21\end{array}$

C Review (and conventions) of two-dimensional supersymmetry 22

$\begin{array}{ll}\text { C.1 }(2,2) & 22\end{array}$

C.2 $(0,2) \quad 24$

D $(2,2)$ and $(0,2)$ supersymmetric backgrounds in superconformal gauge 25

\section{Introduction}

Some $d$-dimensional conformal field theories have exactly marginal operators $\left\{\mathcal{O}_{I}\right\}$. This means that when we add them to the action with coupling constants $\lambda^{I}$,

$$
\delta S=\frac{1}{\pi^{d / 2}} \sum_{I} \int d^{d} x \lambda^{I} \mathcal{O}_{I}(x)
$$

the theory remains conformal. The coefficients $\lambda^{I}$ parameterize the space of conformal field theories, a.k.a. the conformal manifold $\mathcal{M}$. The two-point functions

$$
\left\langle\mathcal{O}_{I}(x) \mathcal{O}_{J}(y)\right\rangle=\frac{g_{I J}\left(\lambda^{K}\right)}{(x-y)^{2 d}}
$$

define a metric, known as the Zamolodchikov metric [1]. It is the metric on the conformal manifold [2]. It carries nontrivial information that cannot be removed by redefinitions of the coupling constants $\lambda^{I}$ [3]. For example, the Ricci scalar associated to $g_{I J}\left(\lambda^{K}\right)$ is invariant under all such redefinitions.

The purpose of this note is to explore the geometry and the topology of $\mathcal{M} .^{1}$ Our main tool will be the conformal anomaly first discussed in [5]. By allowing $\lambda^{I}$ to be

\footnotetext{
${ }^{1}$ In worldsheet string theory, $\mathcal{M}$ can be interpreted as the space of classical vacua of the theory. In the $\mathrm{AdS}_{d+1} / \mathrm{CFT}_{d}$ correspondence, the conformal manifold of the $\mathrm{CFT}_{d}$ is interpreted as the space of vacua in $\mathrm{AdS}_{d+1}$ (see e.g. [4]). These correspondences allow to connect our results to various other topics.
} 
spacetime dependent background fields, [5] derived a contribution to the trace of the energymomentum tensor, which depends on the Zamolodchikov metric $g_{I J}$.

In supersymmetric theories the anomaly above must be supersymmetrized. This introduces a few new elements into the analysis. First, it leads to restrictions on the local form of the metric $g_{I J}$ and it also leads to global restrictions. Second, the anomaly forces us to introduce some contact terms. We will study both aspects in detail.

In section 2 we review the analysis of these conformal anomalies (without supersymmetry). Here we spell out the conditions they have to satisfy and show how a careful analysis leads to new constraints beyond the Wess-Zumino consistency conditions [6].

In the remaining sections we will study $\mathcal{N}=(2,2)$ and $\mathcal{N}=(0,2)$ theories in two dimensions and $\mathcal{N}=2$ theories in four dimensions.

Our discussion of two-dimensional $\mathcal{N}=(2,2)$ theories in section 3 leads to a new proof $^{2}$ that $\mathcal{M}$ factorizes into a space $\mathcal{M}_{c}$ depending on chiral couplings $(\lambda, \bar{\lambda})$ and a space $\mathcal{M}_{t c}$ depending on twisted chiral couplings $(\tilde{\lambda}, \overline{\widetilde{\lambda}})$. Their Kähler potentials are $K_{c}(\lambda, \bar{\lambda})$ and $K_{t c}(\tilde{\lambda}, \overline{\widetilde{\lambda}})$. We will show that $\mathcal{M}$ must be Hodge ${ }^{3}$ and will further argue that its Kähler class should be trivial. This, in particular, shows that $\mathcal{M}$ cannot be a smooth compact manifold.

We will also study the sphere partition function. Without supersymmetry, there are counterterms that render it ambiguous. With $\mathcal{N}=(2,2)$ supersymmetry there are two ways, denoted $A$ and $V$, to place the theory on the sphere and the partition function has universal content. It is given by

$$
Z_{A}=\left(\frac{r}{r_{0}}\right)^{\frac{c}{3}} e^{-K_{c}(\lambda, \bar{\lambda})} ; \quad Z_{V}=\left(\frac{r}{r_{0}}\right)^{\frac{c}{3}} e^{-K_{t c}(\tilde{\lambda}, \overline{\tilde{\lambda}})} .
$$

Here $r$ is the radius of the sphere and $r_{0}$ a (scheme dependent) scale. The dependence on $r$ reflects the ordinary conformal anomaly. As we will show, the appearance of $K_{c}$ or $K_{t c}$ reflects another contribution to the conformal anomaly depending on exactly marginal couplings. The identifications (1.3) were conjectured in [8] and proven in [9] as well as in [10] based on the work of [11-13].

In section 4 we will discuss $\mathcal{N}=(0,2)$ theories in $d=2$. Our analysis leads to restrictions on the metric on $\mathcal{M}$ and shows that $\mathcal{M}$ is Hodge and suggests that its Kähler class is trivial. But we will argue that the two-sphere partition function is not universal.

Section 5 is devoted to $\mathcal{N}=2$ theories in $d=4$. Again, the sphere partition function has universal content and computes the Kähler potential on $\mathcal{M}$

$$
Z=\left(\frac{r}{r_{0}}\right)^{-4 a} e^{K / 12}
$$

This relation was proven in [10] as well as in [14] and was further used in [15-17]. $\mathcal{N}=2$ supersymmetry fixes an additional contribution to the conformal anomaly depending on a four-tensor in $\mathcal{M}$ in terms of the Riemann tensor of the Zamolodchikov metric. As in

\footnotetext{
${ }^{2}$ In this paper we assume that the coupling constants can be promoted to $\mathcal{N}=(2,2)$ chiral and twisted chiral superfields. This assumption is non-trivial as it can fail in some cases [7].

${ }^{3} \mathrm{~A}$ Kähler-Hodge manifold is a Kähler manifold for which the flux of the Kähler two-form through any two-cycle is an integer.
} 
two dimensions, our analysis shows that $\mathcal{M}$ is Hodge and suggests that its Kähler class is trivial. ${ }^{4}$

Our discussion is reminiscent of that of $[19,20]$. In both cases nontrivial contact terms are identified. They cannot be absorbed by supersymmetric local counterterms and therefore correspond to anomalies. They reflect short distance physics and can be analyzed in the flat space theory. Then, these contact terms have interesting consequences when the theory is placed on the sphere.

For a related supersymmetric analysis of conformal anomalies in $\mathcal{N}=1$ theories in $d=4$ and $\mathcal{N}=2$ theories in $d=3$ see $[21,22]$.

Four appendices contain technical results. Appendix A concerns with the normalization of the anomalies. In appendix B we collect some properties of the Fradkin-TseytlinPaneitz-Riegert (FTPR) operator, which appears in the anomaly in $d=4$. Appendix C reviews $(2,2)$ and $(0,2)$ supersymmetry in two dimensions and their linearized supergravities. Appendix D considers $(2,2)$ Poincaré supergravity in superconformal gauge (which always exists locally). We classify the allowed rigid supersymmetric backgrounds in this gauge.

\section{The anomaly associated with the metric on $\mathcal{M}$}

In momentum space the two-point functions (1.2) take the following form

$$
\left\langle\mathcal{O}_{I}(p) \mathcal{O}_{J}(-p)\right\rangle \sim g_{I J}\left\{\begin{array}{cc}
p^{d} & d=2 n+1 \\
p^{2 n} \log \left(\frac{\Lambda^{2}}{p^{2}}\right) & d=2 n .
\end{array}\right.
$$

The explicit scale (or cutoff) $\Lambda$ in the logarithm does not violate scale invariance. The reason is that rescaling $\Lambda$ changes the answer by a polynomial in $p^{2}$, which is a contact term. The correlation function at separated points (1.2) therefore remains intact under rescaling $\Lambda$. Such logarithms appear abundantly in conformal field theories (CFTs). Even though they do not violate the conformal Ward identities, they lead to anomalies (i.e. the non-conservation of the dilatation charge in the presence of non-vanishing background fields). One way to detect it is to make the couplings, $\lambda^{I}, x$-dependent. Then, the trace anomaly in even dimensions includes a term, roughly of the form $[5,23]:^{5}$

$$
T_{\mu}^{\mu} \supset g_{I J} \lambda^{I} \square^{\frac{d}{2}} \lambda^{J}
$$

The precise action of the Laplacian could be to the left and to the right. We will specify this later for $d=2$ and $d=4$.

We study CFTs with spacetime metric $\gamma_{\mu \nu}$ and spacetime dependent coupling constants $\lambda^{I}$. We assume that the theory can be regulated in a diffeomorphism-invariant fashion. Specifically, we assume that the energy-momentum tensor is conserved even at coincident points (apart from the ordinary Ward identity relations). We will be interested in

\footnotetext{
${ }^{4}$ We do not make this claim for $\mathcal{N}=1$ theories in four dimensions. In fact, [18] suggested a construction of compact conformal manifolds in $\mathcal{N}=1$ theories.

${ }^{5}$ This term is in addition to the ordinary conformal anomalies, which depend only on the spacetime metric.
} 
the partition function $Z\left[\gamma_{\mu \nu} ; \lambda^{I}\right]$ and its variation $\delta_{\sigma} \log Z$ under infinitesimal Weyl transformations

$$
\delta_{\sigma} \gamma_{\mu \nu}=2 \delta \sigma \gamma_{\mu \nu}
$$

with infinitesimal $\delta \sigma$ of compact support. Naively, conformal invariance means that the variation vanishes. But because of the anomaly, it does not. This variation satisfies a number of important properties:

1. $Z\left[\gamma_{\mu \nu} ; \lambda^{I}\right]$ is a nonlocal functional of its arguments. However, its variation $\delta_{\sigma} \log Z$ is a local functional of $\gamma_{\mu \nu}, \lambda^{I}$ and $\delta \sigma$.

2. $\delta_{\sigma} \log Z$ must be coordinate invariant in spacetime.

3. It must be coordinate invariant in $\mathcal{M}$. Below we will argue that it should also be globally well defined on $\mathcal{M}$.

4. It must obey the Wess-Zumino consistency condition [6]

$$
\delta_{\sigma_{1}} \delta_{\sigma_{2}} \log Z-\delta_{\sigma_{2}} \delta_{\sigma_{1}} \log Z=0
$$

5. A term in $\delta_{\sigma} \log Z$ that is obtained by a Weyl variation of a local term is considered trivial. An anomaly is a "cohomologically nontrivial" term. It cannot be removed by changing a counterterm. Equivalently, it cannot be removed by changing the renormalization scheme [24]. Therefore, even though the anomaly arises due to a short distance regulator, it is universal - it does not depend on the regularization.

Let us start in $d=2$. The infinitesimal Weyl variation of $\log Z$ responsible for the trace anomaly (2.2) and the ordinary trace anomaly is given by

$$
\delta_{\sigma} \log Z=\frac{c}{24 \pi} \int d^{2} x \delta \sigma \sqrt{\gamma} R-\frac{1}{4 \pi} \int d^{2} x \delta \sigma \sqrt{\gamma} g_{I J} \gamma^{\mu \nu} \partial_{\mu} \lambda^{I} \partial_{\nu} \lambda^{J} .
$$

Here $R$ is the Ricci scalar and the first term is the universal contribution due to the central charge $c$. The normalization of the second term is worked out in appendix A.

The anomaly functional (2.5) includes a sigma model with target space $\mathcal{M}$. It manifestly obeys the Wess-Zumino consistency condition because it is Weyl invariant. There is no local counterterm, whose Weyl variation yields (2.5). Therefore, (2.5) is cohomologically nontrivial. In the language of [25] one could refer to the first term in (2.5) as a type-A and to the second term as a type-B anomaly. ${ }^{6}$

An important part of our discussion will be the analysis of the allowed local counterterms (related to item 5 in the list above). In two dimensions, an important counterterm is

$$
\int d^{2} x \sqrt{\gamma} R F\left(\lambda^{I}\right)
$$

\footnotetext{
${ }^{6} \mathrm{~A}$ type-A anomaly vanishes for $x$-independent $\delta \sigma$ when the background fields have trivial topology. A type-B anomaly does not vanish for constant sigma even for trivial topology and reflects logarithms in certain correlation functions.
} 
Its Weyl variation is

$$
\delta_{\sigma} \int d^{2} x \sqrt{\gamma} R F\left(\lambda^{I}\right)=-2 \int d^{2} x \sqrt{\gamma} \square \delta \sigma F\left(\lambda^{I}\right) .
$$

We will see various consequences of this counterterm in what follows.

In addition to (2.5), there are other potential trace anomalies that we need to consider. First, we have the parity odd type-B anomaly

$$
\delta_{\sigma} \log Z \supset \int d^{2} x \delta \sigma \sqrt{\gamma} B_{I J} \epsilon^{\mu \nu} \partial_{\mu} \lambda^{I} \partial_{\nu} \lambda^{J},
$$

with an anti-symmetric two-form $B_{I J}$ on the conformal manifold $\mathcal{M}$. Also, we have two type-A anomalies

$$
\delta_{\sigma} \log Z \supset \int d^{2} x \partial_{\mu} \delta \sigma \sqrt{\gamma} V_{I} \gamma^{\mu \nu} \partial_{\nu} \lambda^{I}
$$

and

$$
\delta_{\sigma} \log Z \supset \int d^{2} x \partial_{\mu} \delta \sigma \sqrt{\gamma} \widetilde{V}_{I} \epsilon^{\mu \nu} \partial_{\nu} \lambda^{I}
$$

They are characterized by one-forms $V_{I}$ and $\widetilde{V}_{I}$ on $\mathcal{M}$. We note that (2.10) is invariant under the gauge transformation $\widetilde{V}_{I} \rightarrow \widetilde{V}_{I}+\partial_{I} \widetilde{f}$. On the other hand, (2.9) transforms under $V_{I} \rightarrow V_{I}+\partial_{I} f$, but the change is cohomologically trivial. It can be absorbed in the Weyl variation of the local counterterm (2.6) with $F \sim f\left(\lambda^{I}\right)$. This allows us to identify $V_{I}$ and $\widetilde{V}_{I}$ as connections on the conformal manifold $\mathcal{M}$.

We will now show that even though (2.8), (2.9), (2.10) obey the Wess-Zumino consistency conditions, a more detailed analysis leads to further restrictions, ruling out these anomalies. This demonstrates that constraints that go beyond the standard cohomological analysis can further restrict anomalies.

First, a simple argument excludes all type-B anomalies that are beyond that in (2.5). We recall that a type- $\mathrm{B}$ anomaly is associated to a logarithm appearing in a correlation function. Without loss of generality we can study the theory in flat Euclidean spacetime with $\gamma_{\mu \nu}=\delta_{\mu \nu}$. Consider the momentum space correlation function of the exactly marginal operators

$$
\left\langle O_{I_{1}}\left(p_{1}\right) O_{I_{2}}\left(p_{2}\right) \cdots O_{I_{n}}\left(p_{n}\right)\right\rangle=\log \Lambda \delta\left(\sum p_{r}\right) A_{I_{1} I_{2} \cdots I_{n}}+\cdots,
$$

where the ellipses on the right-hand side represent terms independent of the UV cutoff $\Lambda$. Since the operators $O_{I}$ are exactly marginal, the coefficient of the logarithm must be ultra-local, i.e. a polynomial in momentum (otherwise, there would be a beta function for the couplings $\lambda^{I}$ ). Scale invariance constrains $A_{I_{1} I_{2} \cdots I_{n}}$ to be quadratic polynomials in the momenta $p_{r}$. Therefore, we can determine $A_{I_{1} I_{2} \cdots I_{n}}$ by picking specific simple combinations of momenta. For example, for $p_{2}=-p_{1}$ and $p_{3}=p_{4}=\cdots=0$ it is clear that $A_{I_{1} I_{2} \cdots I_{n}} \sim$ $p_{1}^{2} \partial_{I_{3}} \partial_{I_{4}} \cdots \partial_{I_{n}} g_{I_{1} I_{2}}$. Similar other specific cases show that $A_{I_{1} I_{2} \cdots I_{n}}$ is determined entirely by derivatives of the Zamolodchikov metric. This means that the additional parity odd anomaly (2.8) controlled by a two-form cannot be present. More precisely, the argument above shows that $H_{I J K}=\partial_{[I} B_{J K]}=0$ and hence $B_{I J}$ is locally given by $B_{I J}=\partial_{[I} B_{J]}$ for some one-form $B_{J}$. By integration by parts we find that this anomaly is now identical to the type-A anomaly (2.10). We will discuss it below. 
Next, we argue that $V_{I}$ and $\widetilde{V}_{I}$ in the type-A anomalies (2.9), (2.10) must satisfy $\partial_{[I} V_{J]}=\partial_{[I} \widetilde{V}_{J]}=0$, i.e. these connections are flat. These anomalies can be extracted from the following energy-momentum correlator

$$
\left\langle T_{\mu \nu}(z) \mathcal{O}_{I}(x) \mathcal{O}_{J}(y)\right\rangle .
$$

Using the conformal Ward identity at separated points, the correlator must be proportional to $g_{I J}$. There could also be contributions with support at $x=y \neq z$, or $z=x \neq y$, or $z=y \neq x$. In the first case the only contact term allowed by dimensional analysis contains $T_{\mu}^{\mu}$, which has zero separated-points correlation functions. In the second and third case, we can have the contact term $T_{\mu \nu}(x) \mathcal{O}_{I}(0) \sim \delta_{\mu \nu} \delta^{(2)}(x) \mathcal{M}_{I}^{K} \mathcal{O}_{K}(0)$ with some matrix $\mathcal{M}_{I}^{K}$. This would lead to a logarithmic term in the three-point function (2.12), of the type already analyzed above, and hence, it is proportional to the Zamolodchikov metric and does not contribute to the anomalies $V_{I}$ and $\widetilde{V}_{I}$.

Therefore, all the terms in (2.12) that are associated to separated points physics are proportional to $g_{I J}$. They cannot lead to nonzero "field strengths" $\partial_{[I} V_{J]}$ or $\partial_{[I} \widetilde{V}_{J]}$, which are anti-symmetric in $I$ and $J$. Thus, at least locally, the connections $V_{I}$ and $\widetilde{V}_{I}$ are pure gauge and the associated anomalies vanish.

Summarizing, we have seen that even though (2.5), (2.9), (2.10), are a priori allowed anomalies that obey the Wess-Zumino consistency conditions, they can be all excluded. This will have important consequences in what follows.

Thus far we limited ourselves to deformations by exactly marginal operators with coefficients $\lambda^{I}$ as in (1.1). If the CFT also has conserved currents $j_{\mu}^{a}$, then it is natural to couple them to classical background fields $A_{\mu}^{a}$ and examine the anomaly as a function of these fields. The anomaly sigma models now depend on the spacetime metric $\gamma_{\mu \nu}$, the couplings $\lambda^{I}$, and the gauge fields $A_{\mu}^{a}$. The operators $\mathcal{O}_{I}$ are taken to carry charges $-q_{I}^{a}$ and the coupling constants $\lambda^{I}$ thus carry charges $q_{I}^{a}$. Away from $\lambda^{I}=0$ some of the symmetries generated by $j_{\mu}^{a}$ may be thus explicitly broken. Related expressions appear in [23]. In addition to the previous requirements of conformal invariance, coordinate invariance in spacetime and on $\mathcal{M}$, we should now also demand gauge invariance. The equation (2.5) is modified by simply replacing $\partial_{\mu} \lambda^{I} \rightarrow \nabla_{\mu} \lambda^{I}=\partial_{\mu} \lambda^{I}-i q_{J}^{a} A_{\mu}^{a} \lambda^{J}$. In addition, one could encounter new anomalies that contain the field strength $F_{\mu \nu}^{a}$. There could also be 't Hooft anomalies under gauge transformations.

In $d=4$ the local functional that reproduces the logarithm in the two-point function (2.1) is a four-derivative local term. One can construct it by starting with the ansatz $\delta_{\sigma} \log Z \supset \int d^{4} x \delta \sigma \sqrt{\gamma} g_{I J} \square \lambda^{I} \square \lambda^{J}+\cdots$ and covariantize this expression both in spacetime and in $\mathcal{M}$. One also requires that it satisfies the Wess-Zumino consistency condition (2.4). After some work ${ }^{7}$ one finds the expression ${ }^{8}$

$$
\delta_{\sigma} \log Z \supset \frac{1}{192 \pi^{2}} \int d^{4} x \sqrt{\gamma} \delta \sigma\left(g_{I J} \widehat{\square} \lambda^{I} \widehat{\square} \lambda^{J}-2 g_{I J} \partial_{\mu} \lambda^{I}\left(R^{\mu \nu}-\frac{1}{3} \gamma^{\mu \nu} R\right) \partial_{\nu} \lambda^{J}\right) .
$$

\footnotetext{
${ }^{7}$ We use the convention $\left[\nabla_{\mu}, \nabla_{\nu}\right] V_{\rho}=R_{\mu \nu \rho}{ }^{\sigma} V_{\sigma}$.

${ }^{8}$ The fact that the anomaly $\int d^{4} x \sqrt{\gamma} \delta \sigma g_{I J} \partial_{\nu} \lambda^{J} \partial_{\mu} \lambda^{I} R^{\mu \nu}$ is proportional to the Zamolodchikov metric at the fixed point plays a very important role in perturbative proofs of the strong version of the $a$-theorem [5, 26-29]. The situation in $d=6$ unfortunately appears to be more complicated [30, 31]. For a review see [32].
} 
Above $\widehat{\square} \lambda^{I}=\square \lambda^{I}+\Gamma_{J K}^{I} \partial^{\mu} \lambda^{J} \partial_{\mu} \lambda^{K}$, where $\Gamma_{J K}^{I}$ is the usual Christoffel connection on $\mathcal{M}$. The ordinary Laplacian $\square$ is enriched to $\widehat{\square}$ so that the anomaly is coordinate invariant on $\mathcal{M}$, as we demand in general. At this juncture $\Gamma_{J K}^{I}$ could be an arbitrary connection, not necessarily the Levi-Civita one. However, demanding that (2.13) satisfies the WessZumino consistency condition forces $\Gamma_{J K}^{I}$ to be the Levi-Civita connection. Note that (2.13) coincides with expressions that appeared in [28] and [31] in related contexts. The combination (2.13) can be viewed as an interesting variant of the Fradkin-Tseytlin-Paneitz-Riegert operator [33-36], which we discuss further in appendix B.

While we do not present an exhaustive classification of anomalies in four dimensions, there is an additional conformal anomaly that depends on a four-tensor on $\mathcal{M}$ with components $c_{I J K L}$ that we would like to mention:

$$
\delta_{\sigma} \log Z \supset \int d^{4} x \delta \sigma \sqrt{\gamma} c_{I J K L} \partial_{\mu} \lambda^{I} \partial^{\mu} \lambda^{J} \partial_{\nu} \lambda^{K} \partial^{\nu} \lambda^{L} .
$$

The four-tensor $c_{I J K L}$ may be either an independent rank-four tensor on the manifold $\mathcal{M}$, or it may be fixed by the Zamolodchikov metric, e.g. $c_{I J K L} \sim g_{I J} g_{K L}$ or $c_{I J K L} \sim$ $R_{I K J L}+R_{J K I L}$, where $R_{I K J L}$ is the Riemann tensor on $\mathcal{M} .^{9}$ The Wess-Zumino consistency condition (2.4) does not imply a relation between $c_{I J K L}$ and the Zamolodchikov metric. However, in section 5 we will show that in $\mathcal{N}=2$ supersymmetric theories, such a relation must exist, and $c_{I J K L}$ is proportional to the Riemann curvature tensor of the Zamolodchikov metric.

For future references, let us also list some of the allowed counterterms in four dimensions

$$
\int d^{4} x \sqrt{\gamma}\left(R^{2} F_{1}\left(\lambda^{I}\right)+R_{\mu \nu}^{2} F_{2}\left(\lambda^{I}\right)+R_{\mu \nu \rho \sigma}^{2} F_{3}\left(\lambda^{I}\right)+\cdots\right) .
$$

We will be particularly interested in the case where the underlying theory is supersymmetric. Then, the exactly marginal couplings $\lambda^{I}$ reside in various superfields [38]. If the superconformal field theory (SCFT) can be regularized in a supersymmetric manner, then we must further require that the local anomaly functionals above be supersymmetrized. We will study some of the consequences of supersymmetrizing (2.5) and (2.13). We will show that the remaining ambiguity (2.6) in the renormalization scheme in two dimensions and (2.15) in four dimensions is restricted to have holomorphic dependence on the coupling constants. This fact has several important consequences. In particular, it makes the sphere partition function meaningful (up to a Kähler transformation generated by a holomorphic function $[10,14])$.

\section{$3 \mathcal{N}=(2,2)$ supersymmetry in $d=2$}

Our goal in this section is to determine the conformal anomaly and analyze its consequences in $\mathcal{N}=(2,2)$ supersymmetric theories in two dimensions. Here the exactly marginal parameters belong either to background chiral multiplets or twisted chiral multiplets, which we denote by $\lambda^{I}$ and $\widetilde{\lambda}^{A}$, respectively.

\footnotetext{
${ }^{9}$ We thank Y. Nakayama for a discussion on the topic and for stressing the potential relevance of the anomaly (2.14) to the question of locality in $\operatorname{AdS}_{d+1}$. See for instance [37].
} 
First, we should supersymmetrize the anomaly (2.5) and the counterterm (2.6). For that we need to place the theory not only in curved space but in curved superspace [39]. $\mathcal{N}=(2,2)$ supergravity was discussed in [40-44] and, in particular, the possibilities for rigid supersymmetry in curved space were analyzed in [44]. (We repeat this analysis in the superconformal gauge in appendix D.)

We should discuss two distinct supergravity formulations known as $\mathrm{U}(1)_{V}$ and $\mathrm{U}(1)_{A}$ supergravities [40]. These are labeled by whether the U(1) symmetry preserved in the Poincaré supergravity theory is vector or axial. ${ }^{10}$

In terms of the $(2,2)$ SCFT this distinction is the following. The $(2,2)$ SCFT has a $\mathrm{U}(1)_{V} \times \mathrm{U}(1)_{A}$ R-symmetry. We can couple either $\mathrm{U}(1)_{V}$ or $\mathrm{U}(1)_{A}$ to a background gauge field, but an anomaly prevents us from coupling both of them to background fields. Correspondingly, the coincident points divergences and the associated contact terms can preserve either $\mathrm{U}(1)_{V}$ or $\mathrm{U}(1)_{A}$ R-symmetry but not both. These contact terms are described by the corresponding supergravity. Equivalently, we assume that the theory can be regularized while preserving diffeomorphism invariance and supersymmetry as well as either $\mathrm{U}(1)_{V}$ or $\mathrm{U}(1)_{A}$. In particular, we assume that there are no gravitational anomalies so that $c_{L}=c_{R}$.

We find it convenient to use a simplification specific to two dimensions. Since locally every two-dimensional metric is conformally flat, we can describe the metric using the conformal factor $\sigma$ - the Liouville field. This statement is easily supersymmetrized. Every supergravity background can be described locally by a superconformal factor in a superfield. In $\mathrm{U}(1)_{A}$ supergravity it is in a chiral superfield $\Sigma$ and in $\mathrm{U}(1)_{V}$ supergravity it is in a twisted chiral superfield $\widetilde{\Sigma}$ (see appendix $C$ ). The corresponding superconformal variations, whose anomalies we are interested in, are $\delta \Sigma$ and $\delta \widetilde{\Sigma}$ respectively. In what follows we will concentrate mainly on $\mathrm{U}(1)_{A}$. It is straightforward to repeat it for $\mathrm{U}(1)_{V}$.

The supersymmetrization of the conformal anomaly (2.5) is then straightforward. In the regularization preserving $\mathrm{U}(1)_{A}$, the anomaly is given by

$$
\delta_{\Sigma} \log Z_{A}=-\frac{c}{24 \pi} \int d^{2} x d^{4} \theta(\delta \Sigma+\delta \bar{\Sigma})(\Sigma+\bar{\Sigma})+\frac{1}{4 \pi} \int d^{2} x d^{4} \theta(\delta \Sigma \mathcal{K}(\lambda, \bar{\lambda}, \widetilde{\lambda}, \overline{\widetilde{\lambda}})+\text { c.c. }),
$$

where $\mathcal{K}$ is a complex function of the exactly marginal couplings. ${ }^{11}$ Clearly, these expressions obey the Wess-Zumino consistency condition.

One might try to integrate (3.1) to find the $\Sigma$ dependence of $\log Z_{A}$. Although this can be done as a local expression in terms of $\Sigma$, the answer is nonlocal. The point is that it is valid and local in the superconformal gauge, but it is nonlocal in other gauges. This property makes it particularly interesting, as it cannot be absorbed in local counterterms.

\footnotetext{
${ }^{10}$ To follow the discussion below (in our analysis of two-dimensional theories) no familiarity with supergravity is necessary.

${ }^{11}$ In the full supergravity without using the conformal gauge the anomaly takes the form

$$
\delta_{\Sigma} \log Z_{A}=-\frac{c}{24 \pi}\left(\int d^{2} x d^{2} \theta \mathcal{E} \mathcal{R} \delta \Sigma+\text { c.c. }\right)+\frac{1}{4 \pi} \int d^{2} x d^{4} \theta E(\delta \Sigma \mathcal{K}(\lambda, \bar{\lambda}, \tilde{\lambda}, \overline{\tilde{\lambda}})+\text { c.c. })
$$

where $\mathcal{E}$ is the chiral superspace measure, $E$ is the Berezinian superfield, and here $\mathcal{R}$ is a chiral superfield that contains the Ricci scalar in its $\theta^{2}$ component. The first term represents the ordinary anomaly.
} 
In order to proceed we must find the most general supersymmetric expression, which is local in any gauge, and can serve as a local counterterm. This is the supersymmetrization of (2.6). In $\mathrm{U}(1)_{A}$ the local counterterm is [10]

$$
S_{A}=\frac{1}{4 \pi} \int d^{2} x d^{2} \theta \mathcal{R} F(\lambda)+\text { c.c. }=\frac{1}{4 \pi} \int d^{2} x d^{4} \theta \bar{\Sigma} F(\lambda)+\text { c.c. },
$$

where $\mathcal{R}=\bar{D}^{2} \bar{\Sigma}$ is the chiral curvature superfield in superconformal gauge. The counterterm (3.3) depends only on the chiral parameters $\lambda$ and the dependence is holomorphic. ${ }^{12}$ Under a super-Weyl transformation

$$
\delta_{\Sigma} S_{A}=\frac{1}{4 \pi} \int d^{2} x d^{4} \theta(\delta \bar{\Sigma} F(\lambda)+\delta \Sigma \bar{F}(\bar{\lambda})) .
$$

Further restrictions on $\mathcal{K}$ can be found by expanding (3.1) in components and requiring that the forbidden two-dimensional anomalies (2.8), (2.9), (2.10) are absent. Note that this goes beyond the Wess-Zumino consistency conditions. After some algebra, the conclusion is that $\mathcal{K}$ is real and

$$
\mathcal{K}=K_{c}(\lambda, \bar{\lambda})-K_{t c}(\widetilde{\lambda}, \overline{\widetilde{\lambda}})
$$

and therefore the metric on $\mathcal{M}$ is a product metric of two Kähler manifolds $\mathcal{M}=\mathcal{M}_{c} \times \mathcal{M}_{t c}$. The Kähler potential on $\mathcal{M}_{c}$ is $K_{c}$ and it depends only on the chiral parameters and the Kähler potential on $\mathcal{M}_{t c}$ is $K_{t c}$ and it depends only on the twisted chiral parameters. This splitting between the chiral and the twisted chiral parameters is well known and is natural in the context of type II string theory, where $(2,2)$ worldsheet theories lead to $\mathcal{N}=2$ supersymmetry in spacetime. The hypermultiplet and the vector multiplet metrics are factorized as a consequence. Here we see that it follows from properties of anomalies on the worldsheet.

We conclude that the anomaly is

$\delta_{\Sigma} \log Z_{A}=-\frac{c}{24 \pi} \int d^{2} x d^{4} \theta(\delta \Sigma+\delta \bar{\Sigma})(\Sigma+\bar{\Sigma})+\frac{1}{4 \pi} \int d^{2} x d^{4} \theta(\delta \Sigma+\delta \bar{\Sigma})\left(K_{c}(\lambda, \bar{\lambda})-K_{t c}(\widetilde{\lambda}, \overline{\widetilde{\lambda}})\right)$.

Next, we would like to check the invariance of (3.7) under Kähler transformations. It is trivially invariant under

$$
\mathcal{K} \rightarrow \mathcal{K}+G(\widetilde{\lambda})+\bar{G}(\overline{\widetilde{\lambda}}) .
$$

In addition, under the Kähler transformation

$$
\mathcal{K} \rightarrow \mathcal{K}+F(\lambda)+\bar{F}(\bar{\lambda})
$$

the anomaly shifts by the super-Weyl variation (3.5) of the supersymmetric local counterterm (3.3).

\footnotetext{
${ }^{12}$ In the full supergravity without using the conformal gauge the counterterm is

$$
S_{A}=\frac{1}{4 \pi} \int d^{2} x d^{2} \theta \mathcal{E} \mathcal{R} F(\lambda)+\text { c.c. . }
$$
}


The lack of strict Kähler invariance under (3.9) can be interpreted in several different ways with interesting consequences. First, we can simply state that the Kähler transformation should be accompanied by a change in a local counterterm. Second, we can assign a transformation law to $\Sigma$

$$
\Sigma \rightarrow \Sigma+\frac{6}{c} F(\lambda)
$$

and use the first term in (3.7) to achieve full Kähler invariance of the anomaly (here we assume $c \neq 0$ ). This perspective means that $e^{\frac{c}{6} \Sigma}$ is a holomorphic section of a line bundle, whose first Chern class is the cohomology class of the Kähler form on $\mathcal{M}$ and therefore $\mathcal{M}$ must be Hodge. This result, which we have now derived using the anomaly, is known for sigma models with Calabi-Yau target spaces and for general $(2,2)$ theories. It is also natural in the context of string compactification as a property of the four-dimensional supergravity theory [45] (see a refinement of this statement in [46-49]). In that context the action depends on $-\frac{c}{6}(\Sigma+\bar{\Sigma})+\mathcal{K}$ where $\Sigma$ is the spacetime dilaton superfield, or equivalently, it is the spacetime conformal compensator. This is similar to our $\Sigma$, which is the two-dimensional conformal factor. Indeed, integrating the anomaly (3.7) we obtain the anomalous piece of the effective action in superconformal gauge

$$
\log Z_{A} \supset-\frac{c}{48 \pi} \int d^{2} x d^{4} \theta\left(\Sigma+\bar{\Sigma}-\frac{6}{c} \mathcal{K}\right)^{2} .
$$

Finally, we can try to use this analysis to suggest a stronger result. It is well known, and we have used it extensively, that the anomaly variation is a well defined local term. The lack of strict Kähler invariance means that our anomaly is not quite well defined. If the Kähler class of $\mathcal{M}$ is trivial, there is no immediate problem since we are not forced to perform the Kähler transformations (3.9) and we thus have a global description of the theory. Different presentations of the theory might be related by Kähler transformations, but this can be absorbed in a local counterterm or in a redefinition of $\Sigma$. However, when the Kähler class of $\mathcal{M}$ is nontrivial, there is a difficulty. In that case we must cover $\mathcal{M}$ with patches and transition functions that involve Kähler transformations and correspondingly a change in the counterterm (2.6).

Now, consider the couplings changing in spacetime in such a way that we must use the transition functions (e.g. spacetime wraps a nontrivial cycle in $\mathcal{M}$ ). Here different parts of spacetime have coupling constants in different patches in $\mathcal{M}$ and since the transition functions between them need a counterterm, e.g. (2.6), there is no single Lagrangian in all of spacetime! We suggest that such a situation is inconsistent. This would mean that $\mathcal{M}$ and the various fields on it are such that no such transition functions are needed. Therefore, we arrive at the conclusion that $\mathcal{M}$ must have vanishing Kähler class. This argument is analogous to that of [50], with the difference being that we are considering the properties of the space of theories rather than the usual target space of a specific sigma model.

More generally, we should always require that the scale variation of the partition function is a local, globally-defined functional of the background fields. In our context, the anomaly functional contains $K$ explicitly and is therefore not invariant under Kähler transformations. The anomaly functional is well defined only if the Kähler class vanishes. 
Let us now extract some useful physical information from our anomalies (3.7). It suffices for our purposes to evaluate the anomaly keeping only the bottom components of the multiplets of the exactly marginal parameters $\lambda^{I}$ and $\widetilde{\lambda}^{A}$ and of $\delta \Sigma$

$$
\delta \Sigma \mid=\delta \sigma+i \delta a
$$

where $\delta a$ parameterizes the $\mathrm{U}(1)_{V}$ transformation. We find

$$
\begin{aligned}
\delta_{\Sigma} \log Z_{A}=-\frac{1}{2 \pi} \int d^{2} x( & \delta \sigma\left(g_{I \bar{J}} \partial_{\mu} \lambda^{I} \partial^{\mu} \bar{\lambda}^{\bar{J}}+\widetilde{g}_{A \bar{B}} \partial^{\mu} \widetilde{\lambda}^{A} \partial_{\mu} \overline{\widetilde{\lambda}}^{\bar{B}}\right)-\frac{1}{2} \square \delta \sigma K_{c} \\
\quad & \left.\quad \delta a\left(\partial^{\mu} \mathcal{A}_{\mu}+\epsilon^{\mu \nu} \partial_{\mu} \widetilde{\mathcal{A}}_{\nu}\right)+\frac{c}{6}(\delta \sigma \square \sigma+\delta a \square a)\right), \\
g_{I \bar{J}}= & \partial_{I} \partial_{\bar{J}} K_{c}, \\
\widetilde{g}_{A \bar{B}}= & \partial_{A} \partial_{\bar{B}} K_{t c}, \\
\mathcal{A}_{\mu}= & \frac{i}{2}\left(\partial_{I} K_{c} \partial_{\mu} \lambda^{I}-\partial_{\bar{I}} K_{c} \partial_{\mu} \bar{\lambda}^{\bar{I}}\right) \\
\widetilde{\mathcal{A}}_{\mu}= & \frac{i}{2}\left(\partial_{A} K_{t c} \partial_{\mu} \widetilde{\lambda}^{A}-\partial_{\bar{A}} K_{t c} \partial_{\mu} \overline{\widetilde{\lambda}}^{\bar{A}}\right) .
\end{aligned}
$$

Here we have integrated by parts and used the metrics $g_{I \bar{J}}$ and $\widetilde{g}_{A \bar{B}}$ on $\mathcal{M}_{c}$ and $\mathcal{M}_{t c}$ as well as the pull-back of the Kähler one-forms $\mathcal{A}_{\mu}$ and $\widetilde{\mathcal{A}}_{\mu}$, whose exterior derivatives give the Kähler two-forms of $K_{c}$ and $K_{t c}$. We note that only the Kähler potential $K_{c}$ for the chiral multiplets appears in the term proportional to $\square \delta \sigma$ in (3.13). This is due to the fact that $\delta \Sigma$ is a chiral multiplet. This point will be important below.

Another way of stating our equations uses the supercurrent multiplet. As we review in appendix C, the relevant axial supercurrent multiplet consists of real (in Lorentzian signature) $\mathcal{J}_{ \pm \pm}$and a chiral $\mathcal{W}$ satisfying

$$
\bar{D}_{ \pm} \mathcal{J}_{\mp \mp}= \pm D_{ \pm} \mathcal{W}
$$

In a conformal theory $\mathcal{W}=0$. Our anomaly is

$$
\mathcal{W}=-\frac{c}{24 \pi} \mathcal{R}+\frac{1}{4 \pi} \bar{D}^{2}\left(K_{c}(\lambda, \bar{\lambda})-K_{t c}(\widetilde{\lambda}, \overline{\widetilde{\lambda}})\right),
$$

where $\mathcal{R}=\bar{D}^{2} \bar{\Sigma}$ is the chiral curvature superfield. As above, it is invariant under Kähler transformations of $K_{t c}$, but not under Kähler transformations of $K_{c}$. We can absorb Kähler transformations of $K_{c}$ by improvements of the energy-momentum tensor multiplet. Alternatively, we can make it invariant by also shifting $\Sigma$. From the first point of view it thus follows that if the Kähler class of $\mathcal{M}$ is non-vanishing, upon letting the coupling constants wrap some two-cycle in $\mathcal{M}$, we would not be able to define a single energymomentum tensor throughout our two-dimensional space. This again suggests that the Kähler class of $K_{c}$ vanishes.

In the absence of supersymmetry, the last term $\square \delta \sigma K_{c}$ in the first line of (3.13) would be cohomologically trivial and could be tuned away by an appropriate choice of 
regularization scheme. Indeed, this term is proportional to the variation of the local term, $\delta_{\sigma} \int d^{2} x \sqrt{\gamma} R K_{c}$. However, since we are, by assumption, defining the partition function using a supersymmetric regulator, cohomologically trivial terms must arise from the Weyl variation of $\mathrm{U}(1)_{A}$ supergravity invariants. The most general such term is $\delta_{\sigma} \int d^{2} x \sqrt{\gamma} R(F(\lambda)+\bar{F}(\bar{\lambda})$ ) with holomorphic $F(\lambda)$ (see (3.3)). Therefore, modulo Kähler transformations, the anomaly in (3.13) is a genuine new contribution to the trace anomaly in $\mathcal{N}=(2,2)$ SCFTs.

Even in the absence of supersymmetry, the terms in (3.13)

$$
-\frac{1}{2 \pi} \int d^{2} x \delta \sigma\left(g_{I \bar{J}} \partial_{\mu} \lambda^{I} \partial^{\mu} \bar{\lambda}^{\bar{J}}+\widetilde{g}_{A \bar{B}} \partial^{\mu} \widetilde{\lambda}^{A} \partial_{\mu} \widetilde{\lambda}^{B}\right)
$$

are cohomology nontrivial since they cannot be generated by the Weyl variation of any local term. These are precisely the terms we discussed in (2.5). This part of the anomaly is captured by a nonlocal term in the effective action, whose Weyl variation reproduces the anomaly (3.16). Supersymmetry relates this nonlocal universal term to local terms that upon a Weyl transformation give rise to the term $\square \delta \sigma K_{c}$ in (3.13). We can thus reconstruct these terms in the effective action by integrating the anomaly sigma model.

In the evaluation of the partition function for constant sources $\lambda^{I}$ and $\widetilde{\lambda}^{A}$ and vanishing $a$, it suffices to focus on the term $\square \delta \sigma K_{c}$. First we covariantize it

$$
\delta_{\Sigma} \log Z_{A} \supset \frac{1}{4 \pi} \int d^{2} x \sqrt{\gamma} \square \delta \sigma K_{c} .
$$

Using $\delta_{\sigma} \sqrt{\gamma} R=-2 \sqrt{\gamma} \square \delta \sigma$ we learn that the partition function contains

$$
\log Z_{A} \supset-\frac{1}{8 \pi} \int d^{2} x \sqrt{\gamma} R K_{c} .
$$

We repeat that this is not a supersymmetric local term. It is related by supersymmetry to some nonlocal terms that generate the anomaly (3.16). This is the reason the coefficient of (3.18) is physical. The super-Weyl invariant terms in $Z_{A}$ vanish identically since all two-dimensional supergravity backgrounds are superconformally flat.

Upon evaluating the partition function on the two-sphere $S^{2}$ and for constant sources we obtain that the $S^{2}$ partition function of a $\mathcal{N}=(2,2)$ SCFT regularized preserving $\mathrm{U}(1)_{A}$ is

$$
Z_{A}\left[S^{2}\right]=\left(\frac{r}{r_{0}}\right)^{\frac{c}{3}} e^{-\frac{1}{8 \pi} \int_{S^{2}} d^{2} x \sqrt{\gamma} R K_{c}}=\left(\frac{r}{r_{0}}\right)^{\frac{c}{3}} e^{-K_{c}},
$$

where we exhibit the radius of the sphere $r$, which arises from the ordinary central charge anomaly. Note that in agreement with the picture above, we either say that $Z_{A}$ is not Kähler invariant, or we accompany Kähler transformations with $r \rightarrow r e^{\frac{3}{c}(F(\lambda)+\bar{F}(\bar{\lambda}))}$. We remark that (3.19) is correct for any compact manifold with the topology of the two-sphere, the prefactor being reexpressed in terms of the area of the manifold. This is consistent with [9], who argued that the $S^{2}$ partition function is independent of squashing.

The analysis extends almost verbatim in $\mathrm{U}(1)_{V}$ supergravity. In this case the anomaly (3.7) becomes

$$
\delta_{\widetilde{\Sigma}} \log Z_{V} \supset \frac{1}{4 \pi} \int d^{2} x d^{4} \theta(\delta \widetilde{\Sigma}+\delta \overline{\widetilde{\Sigma}})\left(K_{t c}(\lambda, \bar{\lambda})-K_{c}(\widetilde{\lambda}, \overline{\widetilde{\lambda}})\right)
$$


And using $\widetilde{\Sigma}=\sigma+i \widetilde{a}$

$$
\begin{aligned}
\delta_{\widetilde{\Sigma}} \log Z_{V}=-\frac{1}{2 \pi} \int d^{2} x(\delta \sigma & \left(g_{I \bar{J}} \partial_{\mu} \lambda^{I} \partial^{\mu} \bar{\lambda}^{\bar{J}}+\widetilde{g}_{A \bar{B}} \partial^{\mu} \widetilde{\lambda}^{A} \partial_{\mu} \overline{\widetilde{\lambda}}^{\bar{B}}\right)-\frac{1}{2} \square \delta \sigma K_{t c} \\
+ & \left.\delta \widetilde{a}\left(\partial^{\mu} \widetilde{\mathcal{A}}_{\mu}+\epsilon^{\mu \nu} \partial_{\mu} \mathcal{A}_{\nu}\right)+\frac{c}{6}(\delta \sigma \square \sigma+\delta \widetilde{a} \square \widetilde{a})\right) .
\end{aligned}
$$

Now the Kähler potential for the twisted chiral multiplets $K_{t c}$ appears explicitly in the anomaly since $\widetilde{\Sigma}$ is a twisted chiral multiplet. Integrating the anomaly, as above, we arrive at

$$
Z_{V}\left[S^{2}\right]=\left(\frac{r}{r_{0}}\right)^{\frac{c}{3}} e^{-\frac{1}{8 \pi} \int_{S^{2}} d^{2} x \sqrt{\gamma} R K_{t c}}=\left(\frac{r}{r_{0}}\right)^{\frac{c}{3}} e^{-K_{t c}} .
$$

In summary, we have re-derived the result that supersymmetric $S^{2}$ partition functions of $\mathcal{N}=(2,2)$ SCFTs are expressed in terms of the Kähler potential on the appropriate moduli space of theories [8,9] (see also [10]). Our derivation shows that this phenomenon follows directly from a new trace anomaly in supersymmetric field theories. The new trace anomaly is tied by supersymmetry to the anomaly associated with the Zamolodchikov metric. Our methods also led to the suggestion that the Kähler class of $\mathcal{M}$ vanishes.

It is important that the anomalies we discussed reflect UV physics. They are independent of the background spacetime and can be explored locally in flat space. The sphere partition function was used as a tool to extract this anomaly. We note that we simply substituted $\Sigma$ of a sphere.

In appendix D we discuss a classification of supersymmetric backgrounds using our superconformal gauge formalism. Specifically, we consider $\Sigma=\sigma+i a+\theta^{2} w$ with various $\sigma, a$, and $w$. The round sphere discussed above corresponds to $a=0$ but with $\sigma$ and $w$ non-vanishing. We would like now to make a few comments on the other possible supersymmetric backgrounds, and in particular, about the topologically twisted background on the two-sphere $a=i \sigma$ (such that $\Sigma=\overline{\mathcal{R}}=0$ ). Due to the anomaly $c / 6 \int d^{2} x \delta a \square a$ in (3.13), the partition function needs to transform with a nonzero phase under $\mathrm{U}(1)_{V}$ transformations. Hence, the $S^{2}$ partition function on the twisted sphere vanishes (see [51-53]). If one introduces the parameter $\bar{w}$, then this phase can be absorbed by including $\bar{w}^{-c / 3}$ in the partition function. The partition function can be argued to be holomorphic as a function of the coupling constants. But since the holomorphic counterterm (3.3) does not vanish (the anti-holomorphic one vanishes), only the singular part of the dependence on coupling constants is physical. It would be interesting to understand what these singular pieces mean. They were recently computed in $[54,55]$. Another interesting open question concerns Calabi's diastasis, which is a nice Kähler invariant observable in $(2,2)$ SCFTs. It has an elegant interpretation in terms of conformal interfaces [56], and it would be interesting to see if our methods shed light on it. 


\section{$4 \quad(0,2)$ supersymmetric theories}

Here we consider $(0,2)$ SCFTs and study their trace anomaly. Our conventions are such that the supersymmetry is right-moving. The exactly marginal operators are necessarily in Fermi multiplets [57] (see [58] for background on $(0,2)$ models). The corresponding couplings are in chiral superfields $\lambda^{I}$. We will determine their contribution to the conformal anomaly.

We plan to place the theory in a nontrivial supergravity background. This is simpler when the supergravity theory is anomaly free. First, to avoid gravitational anomalies we must relate the left-moving and the right-moving central charges $c_{L}=c_{R}$. Similarly, $c_{R}$ determines the anomaly in the right-moving $\mathrm{U}(1)$ current and we assume that there is also a left-moving $\mathrm{U}(1)$ current with the same anomaly. Then we can gauge an anomaly free linear combination of these two currents. Note that even if we do not have such an anomaly free setup, we can imagine adding decoupled fields to achieve it.

Under these assumptions, the supergravity transformations (which include gauge transformations for a U(1) gauge field) are non-anomalous. One can then naturally couple the theory to the corresponding supergravity and study it in nontrivial supergravity backgrounds. We will refer to the gauged $\mathrm{U}(1)$ symmetry as axial (as in our discussion of $\mathrm{U}(1)_{A}(2,2)$ supergravity above) and then the vector $\mathrm{U}(1)$ symmetry is a global symmetry, suffering from an anomaly.

Before delving into a technical discussion, let us summarize what we find. The trace anomaly in $(0,2)$ models contains a term depending on the Kähler potential, supersymmetrizing the ordinary bosonic anomaly (2.5). But there is an additional term in the trace anomaly that depends on a new function of the couplings, $H$. This function is not fixed by the $(0,2)$ theory. It depends on precisely how we couple the theory to the background fields. It is instructive to consider a $(2,2)$ theory viewed as a $(0,2)$ theory. Then this function $H$ is given as

$$
H \sim K_{c}-K_{t c}
$$

i.e. in this case the function is physical and unambiguous. But in general $(0,2)$ models it is ambiguous. The sphere partition function depends on $H$ and therefore the sphere partition function in such theories is not universal. However, the arguments leading to the conclusion that the Kähler class vanishes do hold in $(0,2)$ models and in particular, the moduli space of SCFTs cannot be compact. This is in accord with intuition from the heterotic string, where $(0,2)$ models are used to construct $\mathcal{N}=1$ supergravity theories in spacetime. In such cases it is known that the vacuum manifold is Kähler-Hodge [45] (see also [46-49]). For a related stringy discussion see [57, 59].

As in the $(2,2)$ theory, we find it convenient to use the superconformal gauge. But unlike the $(2,2)$ theory there are two natural "conformal gauges." The difference between them is in the gauge condition imposed on the $\mathrm{U}(1)$ gauge field.

One possibility is to use the gauge $A_{--}=0$, where $A_{--}$couples to the right-moving $\mathrm{U}(1)$ current $j_{++}$in the superconformal algebra. In this case the remaining degrees of freedom are in a real superfield $V=\sigma+\frac{i}{2} \theta^{+} \Psi_{+}+\frac{i}{2} \bar{\theta}^{+} \bar{\Psi}_{+}+\theta^{+} \bar{\theta}^{+} A_{++}$. In the linearized 
supergravity approximation which we review in appendix C (see also [60]) this corresponds to $\mathcal{H}_{++}=\mathcal{H}_{----}=0$ with $V=\frac{1}{2} \mathcal{H}$.

Alternatively, we impose Lorentz gauge on that gauge field $\partial^{\mu} A_{\mu}=0$, which is solved locally by $A_{\mu}=\epsilon_{\mu \nu} \partial^{\nu} a$. Then the remaining degrees of freedom are in a chiral multiplet $\Sigma=\sigma+i a+i \theta^{+} \Psi_{+}-i \theta^{+} \bar{\theta}^{+} \partial_{++}(\sigma+i a)$.

These two multiplets are almost identical. Given $\Sigma$ we can write $V=\frac{1}{2}(\Sigma+\bar{\Sigma})$. But given $V$, the chiral superfield $\Sigma=-\frac{i}{\partial_{++}} \bar{D}_{+} D_{+} V$ is nonlocal. The lack of locality affects only $a$. Its zero mode is present in $\Sigma$ but not in $V$. Conversely, a constant mode of $A_{++}$ correspond to a linearly growing $a$.

The gauge invariant chiral curvature superfield can be expressed using either of these fields

$$
\mathcal{R}_{-}=2 \partial_{--} \bar{D}_{+} V=\partial_{--} \bar{D}_{+} \bar{\Sigma} .
$$

Super-Weyl transformations are associated with a chiral $\delta \Sigma$ and simply shift $\Sigma$. Their action on $V$ is $2 \delta V=\delta \Sigma+\delta \bar{\Sigma}$. The shift of $a$ by a constant represents the action of the global vector $\mathrm{U}(1)$ symmetry that is not gauged. This global symmetry shifts $\Sigma$ by an imaginary constant and does not act on $V$.

Then, the most general expression for the anomaly action is

$$
\begin{aligned}
\delta_{\Sigma} \log Z= & \frac{c}{12 \pi}\left(i \int d^{2} x d \theta^{+} \delta \Sigma \mathcal{R}_{-}+\text {c.c. }\right) \\
& +\frac{i}{4 \pi} \int d^{2} x d \theta^{+} d \bar{\theta}^{+}\left((\delta \Sigma+\delta \bar{\Sigma}) A_{I} \partial_{--} \lambda^{I}+(\delta \Sigma-\delta \bar{\Sigma}) B_{I} \partial_{--} \lambda^{I}\right)+\text { c.c. } .
\end{aligned}
$$

So far, $A_{I}, B_{I}$ are arbitrary functions of the couplings.

The first term is the ordinary central charge anomaly. It can also be written as follows in our two slightly different versions of conformal gauge:

$$
i \frac{c}{12 \pi} \int d^{2} x d^{2} \theta^{+}(\delta \Sigma-\delta \bar{\Sigma}) \partial_{--}(\Sigma+\bar{\Sigma})=i \frac{c}{6 \pi} \int d^{2} x d^{2} \theta^{+}(\delta \Sigma-\delta \bar{\Sigma}) \partial_{--} V .
$$

The functions $A_{I}, B_{I}$ represent the anomalies that arise in the presence of exactly marginal coupling constants. Already from this we can infer that the metric on $\mathcal{M}$ is Hermitian.

The components expansion of (4.3) leads to a term proportional to $\delta \sigma \epsilon^{\mu \nu}\left(\partial_{\bar{J}} A_{I}-\right.$ $\left.\partial_{I} A_{\bar{J}}\right) \partial_{\nu} \lambda^{I} \partial_{\mu} \bar{\lambda}^{\bar{J}}+$ c.c.. However as we discussed in section 2 , this is consistent only if (locally) $A_{I}=\partial_{I} K$. Furthermore, $K$ has to be real in order to eliminate type-A anomalies that are present upon expanding the first term. Similar considerations show that $B_{I}=\partial_{I} H$ with some real function $H$.

Therefore, the anomaly must be of the form

$$
\begin{aligned}
\delta_{\Sigma} \log Z=\frac{i}{4 \pi} \int d^{2} x d^{2} \theta^{+} & \left((\delta \Sigma-\delta \bar{\Sigma}) \partial_{--}\left(\frac{2 c}{3} V+H\right)\right. \\
& \left.+(\delta \Sigma+\delta \bar{\Sigma})\left(\partial_{I} K \partial_{--} \lambda^{I}-\partial_{\bar{I}} K \partial_{--} \bar{\lambda}^{I}\right)\right) .
\end{aligned}
$$


The expression (4.3) satisfies the usual consistency conditions including the WessZumino conditions. We therefore see that our additional considerations in section 2 concerning which anomalies are allowed show that the metric on $\mathcal{M}$ must be Kähler (in accord with intuition from heterotic compactifications, which lead to $\mathcal{N}=1$ supergravities). Below we will also find some global restrictions on $\mathcal{M}$.

As in (3.14)-(3.15), we can express the anomaly as an operator statement. The theories we study have a supercurrent multiplet with real $R_{ \pm \pm}$and $\mathcal{T}_{----}$satisfying [60] (see appendix C)

$$
\begin{aligned}
\partial_{--} R_{++}+\partial_{++} R_{--} & =0, \\
\bar{D}_{+}\left(\mathcal{T}_{----}-i \partial_{--} R_{--}\right) & =0 .
\end{aligned}
$$

When the theory is conformal we also have $\bar{D}_{+} R_{--}=0 . R_{--}$is the left-moving current that we assumed exists in the CFT. Our anomaly modifies $\bar{D}_{+} R_{--}=0$ to

$$
\bar{D}_{+} R_{--}=i \frac{c}{12 \pi} \mathcal{R}_{-}+\frac{i}{4 \pi} \bar{D}_{+}\left(\partial_{I} K \partial_{--} \lambda^{I}-\partial_{\bar{I}} K \partial_{--} \bar{\lambda}^{\bar{I}}+\partial_{--} H\right)
$$

Next we should identify the ambiguity (i.e. the cohomologically trivial terms that arise from variations of $(0,2)$-supersymmetric local counterterms). This will allow us to determine the actual anomaly. For that we should supersymmetrize (2.6). We can either use the full nonlinear supergravity (see e.g. [61]), or simply use linearized supergravity as in [60] and appendix $\mathrm{C}$ to show that the local counterterm is

$$
-\frac{i}{8 \pi} \int d^{2} x d \theta^{+} h\left(\lambda^{I}\right) \mathcal{R}_{-},
$$

where $h\left(\lambda^{I}\right)$ is holomorphic. This counterterm allows us to absorb some holomorphic transformations on $H$ and $K$ but most of the information in (4.5) is cohomologically nontrivial.

Even though the anomaly associated with $H$ seems like a nontrivial anomaly, which cannot be absorbed in a local counterterm, in fact, it is not physical in $(0,2)$ theories. It can be absorbed in a redefinition of $V .{ }^{13}$ Physically, this means that we redefine the metric and its superpartners by some function of the coupling constants $\lambda^{I}, \bar{\lambda}^{\bar{I}}$. In other words, when we allow the couplings to be general functions, we can add new terms to the Lagrangian that vanish upon setting the couplings to constants. Such a freedom exists in $(0,2)$ theories and it leads to the anomaly $H$. There is no a priori principle that fixes $H$, unless the theory is a $(2,2)$ theory in which case this freedom does not exist and $H$ becomes physical (4.1).

After removing $H$ we conclude that the anomaly can be written as

$$
\delta_{\Sigma} \log Z=i \frac{c}{12 \pi}\left(\int d^{2} x d \theta^{+} \delta \Sigma \mathcal{R}_{-}-\text {c.c. }\right)+\frac{i}{4 \pi} \int d^{2} x d^{2} \theta^{+}(\delta \Sigma+\delta \bar{\Sigma})\left(\partial_{I} K \partial_{--} \lambda^{I}-\text { c.c. }\right) .
$$

\footnotetext{
${ }^{13}$ Note that we cannot absorb $H$ in $\Sigma+\bar{\Sigma}$ by a local transformation. However, this redefinition is indeed a truly local transformation in $(0,2)$ supergravity. It can be understood in linearized supergravity before picking any gauge. There we simply shift $\mathcal{H}$ by the function $H\left(\lambda^{I}, \bar{\lambda}^{\bar{I}}\right)$. This modifies the couplings of the theory to curved space by additional terms in the Lagrangian, which depend on the coupling constants. For conformal theories, this modification only depends on derivatives of the coupling constants. Such ambiguities do not play a role in $(2,2)$ theories.
} 
Expanding it in components with the only nonzero background fields $\Sigma=\sigma+i a$ and $\lambda^{I} \mid$ we find

$$
\begin{aligned}
\delta_{\Sigma} \log Z & =-\frac{1}{2 \pi} \int d^{2} x\left(\frac{c}{6}(\delta \sigma \square \sigma+\delta a \square a)+\delta \sigma G_{I \bar{J}} \partial^{\mu} \lambda^{I} \partial_{\mu} \bar{\lambda}^{\bar{J}}\right. \\
\left.-\frac{1}{4} \square \delta \sigma K+\frac{1}{4} \delta a\left(\partial^{\mu} \mathcal{A}_{\mu}+\epsilon^{\mu \nu} \partial_{\mu} \mathcal{A}_{\nu}\right)\right), & \\
G_{I \bar{J}} & =\partial_{I} \partial_{\bar{J}} K, \quad \\
\mathcal{A}_{\mu} & =i\left(\partial_{I} K \partial_{\mu} \lambda^{I}-\partial_{\bar{I}} K \partial_{\mu} \bar{\lambda}^{\bar{I}}\right) .
\end{aligned}
$$

The first term is the ordinary anomaly. The second term is the anomaly (2.5) associated with the metric on $\mathcal{M}$.

The situation with Kähler transformations is as in $(2,2)$ theories. Kähler transformations can be absorbed in a shift of $\Sigma$. As there, $e^{\frac{c}{6} \Sigma}$ is a holomorphic section of a line bundle over $\mathcal{M}$ and therefore $\mathcal{M}$ is not only Kähler, but it is also Hodge. Also, as in $(2,2)$ theories, we suggest that the Kähler class of $\mathcal{M}$ is in fact trivial.

These results are consistent with the expectation from the string application of these models. When the $(0,2)$ theory is used as the worldsheet of a compactified heterotic string it leads to $\mathcal{N}=1$ supersymmetry in four dimensions and $\mathcal{M}$ is the target space of some of its chiral superfields. In this case it is known that $\mathcal{M}$ should be Kähler $[57,59]$ and Hodge [45]. We extend these conclusions to all $(0,2)$ SCFTs and argue that the Kähler class of $\mathcal{M}$ should be trivial.

While the anomaly functional (4.10) contains the term $\square \delta \sigma K$, the partition function depends on the choice of $H$ (which we have set to zero for simplicity) and therefore it is not universal.

\section{$5 \mathcal{N}=2$ supersymmetry in $d=4$}

We now proceed to the supersymmetric generalization of the conformal anomaly (2.13). For $\mathcal{N}=2$ supersymmetry the appropriate superspace expression is

$$
\delta_{\Sigma} \log Z \supset \frac{1}{192 \pi^{2}} \int d^{4} x d^{4} \theta d^{4} \bar{\theta} E(\delta \Sigma+\delta \bar{\Sigma}) K\left(\lambda^{I}, \bar{\lambda}^{\bar{I}}\right)
$$

The super-Weyl parameters $\delta \Sigma$ and $\delta \bar{\Sigma}$ are chiral and anti-chiral superfields, respectively. They can be viewed as a conformal compensator in $\mathcal{N}=2$ supergravity [62]. $\lambda^{I}$ and $\bar{\lambda}^{\bar{I}}$ are chiral and anti-chiral superfields with Weyl weight zero, whose lowest components are the exactly marginal couplings, which we also denote as $\lambda^{I}$ and $\bar{\lambda}^{\bar{I}} . K(\lambda, \bar{\lambda})$ is the Kähler potential on the conformal manifold $\mathcal{M}$.

In addition to the anomaly that contains the moduli, we also have the usual Weyl anomaly, which depends only on the supergravity multiplet. Its superspace expression is 
an integral over chiral $\mathcal{N}=2$ superspace (see e.g. [63] and appendix B for details)

$$
\begin{aligned}
\delta_{\Sigma} \log Z & \supset \frac{1}{16 \pi^{2}} \int d^{4} x d^{4} \theta \mathcal{E} \delta \Sigma\left(a \Xi+(c-a) W^{\alpha \beta} W_{\alpha \beta}\right)+\text { c.c. } \\
& \supset \frac{1}{16 \pi^{2}} \int d^{4} x \sqrt{\gamma} \delta \sigma\left(c C^{\mu \nu \rho \sigma} C_{\mu \nu \rho \sigma}-a\left(E_{4}-\frac{2}{3} \square R\right)\right),
\end{aligned}
$$

where $W^{\alpha \beta}$ and $\Xi$ are chiral superfields. $W^{\alpha \beta}$ is the Weyl superfield, while $\Xi$ is constructed from curvature superfields that appear in the commutators of super-covariant derivatives in curved superspace.

To work out the component field expansion of (5.1), we need to know the component expansion of an action of the general form

$$
S=\frac{1}{4} \int d^{4} x d^{4} \theta d^{4} \bar{\theta} E \mathcal{K}\left(\lambda^{A}, \bar{\lambda}^{\bar{A}}\right),
$$

where $\lambda^{A}$ and $\bar{\lambda}^{\bar{A}}$ are $\mathcal{N}=2$ chiral and anti-chiral multiplets with Weyl weight $w=0$, respectively. For our anomaly (5.2) we will then specify to

$$
\mathcal{K}\left(\lambda^{A}, \bar{\lambda}^{\bar{A}}\right)=\frac{1}{192 \pi^{2}}(\delta \Sigma+\delta \bar{\Sigma}) K\left(\lambda^{I}, \bar{\lambda}^{\bar{I}}\right) .
$$

For calculating the component expansion of (5.3) we follow [64]. We start with the special case $\mathcal{K}=\mathcal{A} \overline{\mathcal{B}}$, where $\mathcal{A}$ and $\overline{\mathcal{B}}$ are chiral and anti-chiral multiplets respectively. Keeping only the bottom components $\mathcal{A}|=A, \overline{\mathcal{B}}|=\bar{B}$, and the metric background (i.e. dropping the bosonic auxiliary fields in the supergravity multiplet), we get

$$
S=\int d^{4} x \sqrt{\gamma}\left(\nabla^{2} A \nabla^{2} \bar{B}-2 \nabla_{\mu} A\left(R^{\mu \nu}-\frac{1}{3} R \gamma^{\mu \nu}\right) \nabla_{\nu} \bar{B}\right) .
$$

In order to find the answer for a generic $\mathcal{K}\left(\lambda^{A}, \bar{\lambda}^{\bar{A}}\right)$ we expand around a reference point and then use the fact that product of chiral multiplets with Weyl weight $w=0$ is a chiral multiplet with $w=0$ and similarly for anti-chiral multiplets. Then $\mathcal{K}\left(\lambda^{A}, \bar{\lambda}^{\bar{A}}\right)$ can be expressed as a sum $\sum_{i} \mathcal{A}_{i} \overline{\mathcal{B}}_{i}$ with chiral $\mathcal{A}_{i}$ and anti-chiral $\overline{\mathcal{B}}_{i}$ and we can use (5.5) for each term in the sum. Doing this we arrive at

$$
\begin{aligned}
S=\int d^{4} x \sqrt{\gamma}\{ & \mathcal{K}_{A B \overline{C D}} \nabla^{\mu} \lambda^{A} \nabla_{\mu} \lambda^{B} \nabla^{\nu} \bar{\lambda}^{\bar{C}} \nabla_{\nu} \bar{\lambda}^{\bar{D}}+\mathcal{K}_{A B \bar{C}} \nabla^{\mu} \lambda^{A} \nabla_{\mu} \lambda^{B} \square \bar{\lambda}^{\bar{C}} \\
& +\mathcal{K}_{\overline{A B} C} \nabla^{\mu} \bar{\lambda}^{\bar{A}} \nabla_{\mu} \bar{\lambda}^{\bar{B}} \square \lambda^{C}+\mathcal{K}_{A \bar{B}} \square \lambda^{A} \square \bar{\lambda}^{\bar{B}} \\
& \left.-2 \mathcal{K}_{A \bar{B}} \nabla_{\mu} \lambda^{A}\left(R^{\mu \nu}-\frac{1}{3} R \gamma^{\mu \nu}\right) \nabla_{\nu} \bar{\lambda}^{\bar{B}}\right\}
\end{aligned}
$$

where $\lambda^{A}=\left(\Sigma, \lambda^{I}\right)\left(\bar{\lambda}^{\bar{A}}=\left(\bar{\Sigma}, \bar{\lambda}^{\bar{I}}\right)\right.$. Using (5.4) and the following definitions for the metric, connection and curvature on a Kähler manifold with Kähler potential $K$

$$
\begin{aligned}
g_{I \bar{J}} & =\partial_{I} \partial_{\bar{J}} K \\
\Gamma_{J K}^{I} & =g^{I \bar{L}} \partial_{J} \partial_{K} \partial_{\bar{L}} K \\
\mathcal{R}_{I \bar{J} K \bar{L}} & =\partial_{I} \partial_{\bar{J}} g_{K \bar{L}}-g^{M \bar{N}} \partial_{I} g_{K \bar{L}} \partial_{\bar{J}} g_{M \bar{N}},
\end{aligned}
$$


we arrive, after several integrations by parts, at

$$
\begin{aligned}
\delta_{\Sigma} & \log Z \supset \frac{1}{96 \pi^{2}} \int d^{4} x \sqrt{\gamma}\left\{\delta \sigma \mathcal{R}_{I \bar{K} J} \nabla^{\mu} \lambda^{I} \nabla_{\mu} \lambda^{J} \nabla^{\nu} \bar{\lambda}^{K} \nabla_{\nu} \bar{\lambda}^{\bar{L}}\right. \\
& +\delta \sigma g_{I \bar{J}}\left(\widehat{\square} \lambda^{I} \hat{\square} \bar{\lambda}^{\bar{J}}-2\left(R^{\mu \nu}-\frac{1}{3} R \gamma^{\mu \nu}\right) \nabla_{\mu} \lambda^{I} \nabla_{\nu} \bar{\lambda}^{\bar{J}}\right) \\
& +\frac{1}{2} K \square^{2} \delta \sigma+\frac{1}{6} K \nabla^{\mu} R \nabla_{\mu} \delta \sigma+K\left(R^{\mu \nu}-\frac{1}{3} \gamma^{\mu \nu} R\right) \nabla_{\mu} \nabla_{\nu} \delta \sigma \\
& -2 g_{I \bar{J}} \nabla^{\mu} \lambda^{I} \nabla^{\nu} \bar{\lambda}^{\bar{J}} \nabla_{\mu} \nabla_{\nu} \delta \sigma+i g_{I \bar{J}}\left(\widehat{\nabla}^{\mu} \widehat{\nabla}^{\nu} \lambda^{I} \nabla_{\nu} \bar{\lambda}^{\bar{J}}-\widehat{\nabla}^{\mu} \widehat{\nabla}^{\nu} \bar{\lambda}^{\bar{J}} \nabla_{\nu} \lambda^{I}\right) \nabla_{\mu} \delta a \\
& -\frac{i}{2}\left(\widehat{\nabla}_{I} \widehat{\nabla}_{J} K \nabla^{\mu} \lambda^{I} \nabla_{\mu} \lambda^{J}-\widehat{\nabla}_{\bar{I}} \widehat{\nabla}_{\bar{J}} K \nabla^{\mu} \bar{\lambda}^{\bar{I}} \nabla_{\mu} \bar{\lambda}^{\bar{J}}+\nabla_{I} K \widehat{\square} \lambda^{I}-\nabla_{\bar{I}} K \widehat{\square} \bar{\lambda}^{\bar{I}}\right) \square \delta a \\
& \left.+i\left(R^{\mu \nu}-\frac{1}{3} R \gamma^{\mu \nu}\right)\left(\nabla_{I} K \nabla_{\mu} \lambda^{I}-\nabla_{\bar{I}} K \nabla_{\mu} \bar{\lambda}^{\bar{I}}\right) \nabla_{\nu} \delta a\right\} .
\end{aligned}
$$

As in (2.13), the hats denote covariant derivatives with respect to target space diffeomorphisms acting on the $\lambda^{I}$. Note that this action is completely covariant under target space diffeomorphisms and we nicely identify the term (2.13) in the second line of (5.8). We also identify the new anomaly (2.14) in the first line. It appears with the Riemann tensor of $\mathcal{M}$. To take into account the complete anomaly we have to add (5.2) to (5.8).

Using the expressions in appendix B one realizes that the terms in the third line of (5.8) can be written as a variation of a local term. Specifically,

$$
\begin{aligned}
\sqrt{\gamma}\left(\frac{1}{2} \square^{2} \delta \sigma+\right. & \left.\frac{1}{6} \nabla^{\mu} R \nabla_{\mu} \delta \sigma+\left(R^{\mu \nu}-\frac{1}{3} \gamma^{\mu \nu} R\right) \nabla_{\mu} \nabla_{\nu} \delta \sigma\right)=\sqrt{\gamma} \frac{1}{2} \Delta_{4} \delta \sigma \\
& =\delta_{\sigma}\left(\sqrt{\gamma}\left[\frac{1}{8} E_{4}-\frac{1}{12} \square R+c C^{2}\right]\right),
\end{aligned}
$$

where $c$ is an arbitrary function of the moduli and $\Delta_{4}$ the FTPR operator (see appendix B). For $c=0$ this is precisely the combination that appears in the $\mathcal{N}=2$ supersymmetric version of the Gauss-Bonnet invariant (see e.g. [65]). The supersymmetric Gauss-Bonnet term may include as a prefactor an arbitrary holomorphic function of the moduli. Therefore, (5.8) is cohomologically trivial if $K=F+\bar{F}$ is a sum of a holomorphic and antiholomorphic function of the moduli, in which case it reduces to

$$
\frac{1}{192 \pi^{2}} \int d^{4} x \sqrt{\gamma}\left(F \Delta_{4} \bar{\sigma}+\text { c.c. }\right) \text {. }
$$

Indeed, consider the following local superspace counterterm

$$
\int d^{4} x d^{4} \theta \mathcal{E} F(\lambda)\left(\Xi-W^{\alpha \beta} W_{\alpha \beta}\right)+\text { c.c. }
$$

The combination $\Xi-W^{\alpha \beta} W_{\alpha \beta}$ contains the Euler combination $E_{4}-\frac{2}{3} \square R$ and its Weyl variation is the supersymmetrization of (5.10).

To arrive at the $S^{4}$ partition function we simply need to integrate the combination appearing in (5.9) on $S^{4}$. Using

$$
\int_{S^{4}} \sqrt{\gamma}\left(E_{4}-\frac{2}{3} \square R+c C^{2}\right)=64 \pi^{2}
$$


we find ${ }^{14}$

$$
Z\left[S^{4}\right]=\left(\frac{r}{r_{0}}\right)^{-4 a} e^{K / 12},
$$

as claimed in [10] and [14]. We note that (5.13) is true for any superconformally flat compact four-manifold if we express the prefactor in terms of its volume.

The Kähler ambiguity $K \rightarrow K+F+\bar{F}$ of the partition function is taken care of by the ambiguous local counterterm (5.11). As in $d=2$, we will now see that the trace anomaly is invariant under a correlated Kähler shift and Weyl transformation.

To find the change of the anomaly polynomial (5.2) under an infinitesimal Weyl transformation $\delta \widetilde{\Sigma}$, we use (cf. [63])

$$
\delta_{\widetilde{\Sigma}} \Xi=2 \delta \widetilde{\Sigma} \Xi-2 \bar{\Delta} \delta \overline{\widetilde{\Sigma}}
$$

where $\bar{\Delta}$, the chiral projection operator, is the $\mathcal{N}=2$ supersymmetric generalization of the FTPR operator (see also appendix B). The Weyl superfield $W^{\alpha \beta}$ transforms homogeneously with weight one, while the chiral superspace density $\mathcal{E}$ transforms with weight -2 (the full superspace density $E$ is invariant). We then find

$$
\delta_{\widetilde{\Sigma}} \delta_{\Sigma} \log Z=-\frac{a}{8 \pi^{2}} \int d^{4} x d^{4} \theta \mathcal{E} \delta \Sigma \bar{\Delta} \delta \overline{\widetilde{\Sigma}}+\text { c.c. . }
$$

On the other hand, under a Kähler shift $K \rightarrow K+F+\bar{F}$ it transforms as

$$
\delta_{F} \delta_{\Sigma} \log Z=\frac{1}{192 \pi^{2}} \int d^{4} x d^{8} \theta E(\delta \Sigma \bar{F}+\delta \bar{\Sigma} F)=\frac{1}{192 \pi^{2}} \int d^{4} x d^{4} \theta \mathcal{E} \delta \Sigma \bar{\Delta} \bar{F}+\text { c.c. }
$$

Therefore, choosing

$$
\delta \widetilde{\Sigma}=\frac{1}{24 a} F,
$$

the anomaly polynomial is invariant under an infinitesimal joint Kähler-Weyl transformation and therefore also under a finite transformation. The invariance can be explicitly seen to hold for the partition function (5.13).

As in two dimensions, this means that $\mathcal{M}$ is not only Kähler, but it is also Hodge. In addition, using background $\lambda^{I}$ that vary in spacetime and wrap a nontrivial cycle in $\mathcal{M}$, we argue that the Kähler class of $\mathcal{M}$ must be trivial. (For certain cases with an $\mathcal{N}=4$ $\mathrm{AdS}_{5}$ dual, it has been argued in [66] that $\mathcal{M}$ is special-Kähler. It would be interesting to understand when this happens in general.)

\section{Acknowledgments}

We would like to thank C. Bachas, C. Closset, S. Cremonesi, L. Di Pietro, N. Ishtiaque, S. Kuzenko, D. Morrison, Y. Nakayama, V. Niarchos, H. Ooguri, H. Osborn, K. Papadodimas, K. Skenderis, E. Witten, and J. Zhou for useful discussions. Z.K. would like to thank the Perimeter Institute for its very kind hospitality during the course of this project. The work of NS was supported in part by DOE grant DE-SC0009988. Z.K. is supported by

\footnotetext{
${ }^{14}$ Since the $S^{4}$ background is superconformally flat, the super-Weyl invariant terms in $Z$ vanish.
} 
the ERC STG grant 335182, by the Israel Science Foundation under grant $884 / 11$, by the United States-Israel Bi- national Science Foundation (BSF) under grant 2010/629 as well as by the Israel Science Foundation center for excellence grant (grant no. 1989/14). A.S. and Z.K. are supported by the I-CORE Program of the Planning and Budgeting Committee. P.H. is supported by Physics Department of Princeton University. A.S. and S.T. acknowledge support from GIF - the German-Israeli Foundation for Scientific Research and Development. This research was supported in part by Perimeter Institute for Theoretical Physics. Research at Perimeter Institute is supported by the Government of Canada through Industry Canada and by the Province of Ontario through the Ministry of Research and Innovation. J.G. also acknowledges further support from an NSERC Discovery Grant and from an ERA grant by the Province of Ontario. Any opinions, findings, and conclusions or recommendations expressed in this material are those of the authors and do not necessarily reflect the views of the funding agencies.

\section{A Normalization of the anomaly}

The normalization of the anomaly (2.5) and (2.13) is fixed as follows. We compute the change of the contact term in $d=2$ under constant rescaling of the coordinates $x \rightarrow e^{\lambda} x$ by writing the two-point function as

$$
\frac{1}{|x|^{4}}=\frac{1}{32} \square^{2}\left(\log ^{2}\left(x^{2} \mu^{2}\right)\right)
$$

whose anomalous Weyl variation is

$$
\delta_{\lambda}^{(\text {anom })} \frac{1}{|x|^{4}}=\frac{\lambda \pi}{2} \square \delta^{(2)}(x) .
$$

This is the contact term that is reproduced by the anomaly functional (2.5).

In $d=4$ we write

$$
\frac{1}{|x|^{8}}=-\frac{1}{768} \square^{3}\left(\frac{\log \left(x^{2} \mu^{2}\right)}{x^{2}}\right)
$$

whose anomalous Weyl variation is

$$
\delta_{\lambda}^{(\text {anom })} \frac{1}{|x|^{8}}=\frac{\lambda \pi^{2}}{96} \square^{2} \delta^{(4)}(x) .
$$

This is matched by the anomaly (2.13).

\section{B The FTPR operator and its properties}

We collect some properties of the Fradkin-Tseytlin-Paneitz-Riegert operator [33-36]. It arises in our context in the case that there is only one exactly marginal modulus and one integrates (2.13) by parts. One obtains, up to cohomologically trivial terms, that the anomaly is

$$
\int d^{4} x \sqrt{\gamma} \delta \sigma \lambda \Delta_{4} \lambda
$$


where

$$
\Delta_{4}=\square^{2}+\frac{1}{3} \nabla^{\mu} R \nabla_{\mu}+2 R^{\mu \nu} \nabla_{\mu} \nabla_{\nu}-\frac{2}{3} R \square
$$

is the FTPR-operator with the defining property that under Weyl rescaling of the metric,

$$
\Delta_{4} \rightarrow e^{-4 \delta \sigma} \Delta_{4}
$$

when it acts on a scalar. Another property of $\Delta_{4}$ which is used in section 5 is

$$
\delta_{\sigma}\left(E_{4}-\frac{2}{3} \square R\right)=-4 \delta \sigma\left(E_{4}-\frac{2}{3} \square R\right)+4 \Delta_{4} \delta \sigma .
$$

This can be derived using

$$
\begin{aligned}
\delta_{\sigma} R & =-2 \delta \sigma R-6 \square \delta \sigma, \\
\delta_{\sigma} R_{\mu \nu} & =-2 \nabla_{\mu} \nabla_{\nu} \delta \sigma-g_{\mu \nu} \square \delta \sigma, \\
\delta_{\sigma} \square R & =-4 \delta \sigma \square R-2 R \square \delta \sigma-2 \nabla^{\mu} R \nabla_{\mu} \delta \sigma-6 \square^{2} \delta \sigma,
\end{aligned}
$$

and the expression for the Euler density which we normalize to

$$
E_{4}=C^{\mu \nu \rho \sigma} C_{\mu \nu \rho \sigma}-2 R^{\mu \nu} R_{\mu \nu}+\frac{2}{3} R^{2}
$$

such that

$$
\int_{S^{4}} d^{4} x \sqrt{\gamma} E_{4}=64 \pi^{2}
$$

Here $C_{\mu \nu \rho \sigma}$ is the Weyl tensor.

In two dimensions instead of $\Delta_{4}$ we have $\Delta_{2} \equiv \square$. It satisfies $\Delta_{2} \rightarrow e^{-2 \delta \sigma} \Delta_{2}$ and $\delta E_{2}=\delta R=-2 \delta \sigma R-2 \Delta_{2} \delta \sigma$ with $\int_{S^{2}} d^{2} x \sqrt{\gamma} E_{2}=8 \pi$.

The conformally covariant operators have generalizations in chiral superspace. In $d=2, \mathcal{N}=(2,2)$ this is the chiral projection operator $\bar{\nabla}^{2}$ which transforms as $\bar{\nabla}^{2} \rightarrow e^{\delta \Sigma} \bar{\nabla}^{2}$ under super-Weyl transformations, which are parameterized by a chiral superfield $\delta \Sigma$ (with a similar transformation for the anti-chiral projector $\nabla^{2}$ ).

For $\mathcal{N}=2$ in $d=4$ the analog of the FTPR operator is the chiral projection operator $\bar{\Delta}$ with the infinitesimal transformation $\delta_{\Sigma} \bar{\Delta}=2 \delta \Sigma \bar{\Delta}$ under a super-Weyl transformation parameterized by a chiral scalar superfield $\delta \Sigma$. Its precise definition in terms of supercovariant derivatives and curvature superfields is reviewed in [63], where one also finds references to the original literature.

\section{Review (and conventions) of two-dimensional supersymmetry}

\section{C.1 $(2,2)$}

We will use the notation $x^{ \pm \pm}$for the coordinates, which makes it easier to compare with spinors. In Euclidean signature $x^{++}$is the complex conjugate of $x^{--}$and in Lorentzian signature they are two real independent coordinates. The Ricci scalar is given by $R \sqrt{\gamma}=$ $-\frac{1}{2} \square \log \gamma$ in the conformal gauge, where $\gamma=\operatorname{det} \gamma_{\mu \nu}$. 
The supercovariant derivatives, which can be obtained from the four-dimensional ones of Wess and Bagger by dimensional reduction, are

$$
D_{ \pm}=\frac{\partial}{\partial \theta^{ \pm}}-i \bar{\theta}^{ \pm} \partial_{ \pm \pm}, \quad \bar{D}_{ \pm}=-\frac{\partial}{\partial \bar{\theta}^{ \pm}}+i \theta^{ \pm} \partial_{ \pm \pm},
$$

The algebra is

$$
\left\{D_{+}, \bar{D}_{+}\right\}=2 i \partial_{++}, \quad\left\{D_{-}, \bar{D}_{-}\right\}=2 i \partial_{--} .
$$

Chiral superfields $\lambda$ and twisted chiral superfields $\tilde{\lambda}$ are defined by

$$
\begin{aligned}
& \bar{D}_{ \pm} \lambda=0 \\
& \bar{D}_{+} \widetilde{\lambda}=D_{-} \widetilde{\lambda}=0 .
\end{aligned}
$$

There are two interesting energy-momentum supermultiplets in a $(2,2)$ theory with an R-symmetry [60]. They are related by mirror symmetry. First, there is the $\mathrm{U}(1)_{V}$ supermultiplet

$$
\bar{D}_{ \pm} \mathcal{R}_{\mp \mp}= \pm \chi_{\mp}, \quad \bar{D}_{+} \chi_{ \pm}=\bar{D}_{-} \chi_{ \pm}=0, \quad D_{+} \chi_{-}=\bar{D}_{-} \bar{\chi}_{+} .
$$

It immediately follows that the bottom component, $\mathcal{R}_{\mp \mp} \mid \equiv j_{\mp \mp}^{V}$ is a conserved vector current

$$
\partial_{++} j_{--}^{V}+\partial_{--} j_{++}^{V}=0 .
$$

Often, there exists a twisted chiral operator $\widetilde{T}$ such that

$$
\chi_{+}=\bar{D}_{+} \overline{\widetilde{T}}, \quad \chi_{-}=-\bar{D}_{-} \widetilde{T}
$$

and then the last two equations in (C.4) are automatically satisfied and the first becomes

$$
\bar{D}_{+} \mathcal{R}_{--}=-\bar{D}_{-} \widetilde{T}, \quad \bar{D}_{-} \mathcal{R}_{++}=-\bar{D}_{+} \overline{\widetilde{T}} .
$$

The $\mathrm{U}(1)_{A}$ supermultiplet is obtained formally by acting with a mirror symmetry transformation on (C.4)

$$
\begin{aligned}
\bar{D}_{ \pm} \mathcal{J}_{\mp \mp} & = \pm \mathcal{Y}_{\mp}, \quad D_{ \pm} \mathcal{Y}_{ \pm}=0, \quad \bar{D}_{ \pm} \mathcal{Y}_{\mp}=0, \\
D_{+} \mathcal{Y}_{-}+D_{-} \mathcal{Y}_{+} & =0 .
\end{aligned}
$$

In this case $j_{\mp \mp}^{A} \equiv \mathcal{J}_{\mp \mp} \mid$ is a conserved axial current

$$
\partial_{++} j_{--}^{A}-\partial_{--} j_{++}^{A}=0 .
$$

Often, there is a chiral $\mathcal{W}$ such that

$$
\mathcal{Y}_{ \pm}=D_{ \pm} \mathcal{W}
$$

and then (C.8) is replaced by

$$
\bar{D}_{ \pm} \mathcal{J}_{\mp \mp}= \pm D_{\mp} \mathcal{W}
$$


Now we discuss linearized coupling to supergravity (see also the analysis of [44].) We start from the case of $\mathrm{U}(1)_{A}$ supergravity. The supergravity multiplet is $\left(\mathcal{H}_{ \pm \pm}, \Sigma\right)$, where $\mathcal{H}_{ \pm \pm}$is real (in Lorentzian signature) and $\Sigma$ is chiral. The linearized coupling to matter then takes the form

$$
\delta \mathcal{L}=\int d^{4} \theta \sum_{ \pm} \mathcal{H}_{ \pm \pm} \mathcal{J}_{\mp \mp}+\left(\int d^{2} \theta \Sigma \mathcal{W}+\text { c.c. }\right)
$$

This is invariant under the linearized transformations

$$
\begin{aligned}
\delta \mathcal{H}_{ \pm \pm} & =D_{ \pm} \bar{L}_{ \pm}-\bar{D}_{ \pm} L_{ \pm}, \\
\delta \Sigma & =-\bar{D}^{2}\left(D_{+} L_{-}-D_{-} L_{+}\right),
\end{aligned}
$$

where $L_{ \pm}$are arbitrary superfields. Note that the first line of (C.13) is consistent with the reality of $\mathcal{H}_{ \pm \pm}$and that the action (C.12) is invariant under (C.13) by using the defining relations (C.4).

In the superconformal gauge $\mathcal{H}_{ \pm \pm}=0$ and the only degree of freedom is $\Sigma=\sigma+i a+\ldots$. Here $\sigma$ is the conformal factor in the metric and $a$ represents the $\mathrm{U}(1)_{A}$ gauge field in Lorentz gauge $A_{\mu}=\epsilon_{\mu \nu} \partial^{\nu} a$. Under the super-Weyl transformations $\Sigma$ is shifted by a chiral superfield $\delta \Sigma$. The shift of $a$ by a constant is a $\mathrm{U}(1)_{V}$ global symmetry transformation.

The situation in $\mathrm{U}(1)_{V}$ supergravity is analogous. In that case the only mode in the superconformal gauge is the twisted chiral superfield $\widetilde{\Sigma}$.

\section{C.2 $(0,2)$}

For $(0,2)$ theories with a conserved $R$-symmetry the supercurrent multiplet consists of three superfields, $R_{ \pm \pm}, \mathcal{T}_{----}$, which are real in Lorentzian signature. They satisfy [60]

$$
\begin{aligned}
\partial_{--} R_{++}+\partial_{++} R_{--} & =0, \\
\bar{D}_{+}\left(\mathcal{T}_{----}-i \partial_{--} R_{--}\right) & =0 .
\end{aligned}
$$

Hence, the R-current is vectorial $\partial_{++} j_{--}+\partial_{--} j_{++}=0$. In components we have

$$
\begin{aligned}
R_{++} & =j_{++}-i \theta^{+} S_{+++}-i \bar{\theta}^{+} \bar{S}_{+++}-\theta^{+} \bar{\theta}^{+} T_{++++}, \\
R_{--} & =j_{--}-i \theta^{+} S_{+--}-i \bar{\theta}^{+} \bar{S}_{+--}-\theta^{+} \bar{\theta}^{+} T_{++--}, \\
\mathcal{T}_{----} & =T_{----}-\theta^{+} \partial_{--} S_{+--}+\bar{\theta}^{+} \partial_{--} \bar{S}_{+--}-\theta^{+} \bar{\theta}^{+} \partial_{--} \partial_{++} j_{--} .
\end{aligned}
$$

We couple this theory to linearized supergravity in a standard fashion. We introduce three real superfields, $\mathcal{H}_{++}, \mathcal{H}, \mathcal{H}_{----}$, and the linearized coupling takes the form

$$
\mathcal{L}=\int d \theta^{+} d \bar{\theta}^{+}\left(\mathcal{T}_{----} \mathcal{H}_{++}+R_{--} \mathcal{H}+R_{++} \mathcal{H}_{----}\right)
$$

The complete super-diffeomorphism group is generated by the following transformations

$$
\begin{aligned}
\mathcal{H}_{++} & \rightarrow \mathcal{H}_{++}+\left(\Lambda_{++}+\bar{\Lambda}_{++}\right), \\
\mathcal{H} & \rightarrow \mathcal{H}+i \partial_{--}\left(\Lambda_{++}-\bar{\Lambda}_{++}\right)+\partial_{++} U_{--}, \\
\mathcal{H}_{----} & \rightarrow \mathcal{H}_{----}+\partial_{--} U_{--} .
\end{aligned}
$$

Above $\Lambda$ is chiral and $\bar{\Lambda}$ is anti-chiral. $U$ is a general multiplet. 
The curvature is in the invariant chiral superfield

$$
\begin{aligned}
\mathcal{R}_{-} & =\bar{D}_{+}\left(i \partial_{--}^{2} \mathcal{H}_{++}+\partial_{--} \mathcal{H}-\partial_{++} \mathcal{H}_{----}\right) \\
& =-i\left(\partial_{--} \bar{\Psi}_{+}-\partial_{++} \bar{\Psi}_{---}\right)-\frac{i}{4} \theta^{+}(R \sqrt{\gamma}-2 i F) .
\end{aligned}
$$

Here $\Psi_{+}$and $\Psi_{---}$are components of the "gravitino" and originate from $\mathcal{H}$ and $\mathcal{H}$ respectively. $R$ and $F$ are the Ricci scalar and the field strength of the U(1) R-gauge field, respectively.

\section{D $(2,2)$ and $(0,2)$ supersymmetric backgrounds in superconformal gauge}

In this appendix we repeat the classification of supersymmetric backgrounds of [44]. These authors used linearized supergravity to find the equations for supersymmetric backgrounds and then covariantized them. Instead, we will use the superconformal gauge. This way we will not have to rely on linearized or the full nonlinear supergravity. The point is that every $(2,2)$ background in two dimensions can be brought locally to a superconformal gauge and then all the information is contained in a chiral superfield $\Sigma$. (More precisely, we will be using $\mathrm{U}(1)_{A}$ supergravity where the conformal factor is in a chiral multiplet. It is trivial to repeat the analysis in $\mathrm{U}(1)_{V}$.)

As we said, the advantage of using this presentation is that there is no need to use supergravity. We simply use flat space ordinary superconformal symmetry.

We will set the fermionic components of $\Sigma$ to zero; i.e. $\Sigma=\sigma+i a+\theta^{2} w, \bar{\Sigma}=\sigma-i a+\bar{\theta}^{2} \bar{w}$. We will assume that $\sigma$, which is the conformal factor, is real. But we will allow non-unitary backgrounds in which $a$ can be complex and $\bar{w}$ is not the complex conjugate of $w$.

Most of our analysis will be local. The global considerations are easily implemented later. We will denote the Killing spinors for the supersymmetry variation as $\zeta^{\alpha}, \bar{\zeta}^{\alpha}$ with $\alpha= \pm$, and will view them as four independent complex variables (no particular reality). The conditions for supersymmetry are

$$
\begin{array}{rlrl}
\partial_{ \pm \pm} \zeta^{\mp}=\partial_{ \pm \pm} \bar{\zeta}^{\mp} & =0 \\
\partial_{++}\left(e^{\sigma+i a} \bar{\zeta}^{+}\right)+\frac{i}{2} w e^{\sigma+i a} \zeta^{-} & =0, & & \partial_{--}\left(e^{\sigma+i a} \bar{\zeta}^{-}\right)-\frac{i}{2} w e^{\sigma+i a} \zeta^{+}=0 \\
\partial_{++}\left(e^{\sigma-i a} \zeta^{+}\right)+\frac{i}{2} \bar{w} e^{\sigma-i a} \bar{\zeta}^{-} & =0, & \partial_{--}\left(e^{\sigma-i a} \zeta^{-}\right)-\frac{i}{2} \bar{w} e^{\sigma-i a} \bar{\zeta}^{+}=0 .
\end{array}
$$

The first equation is the standard restriction due to flat space superconformal symmetry. The remaining equations state that the fermionic components of $e^{\Sigma}$ and $e^{\bar{\Sigma}}$ are invariant. Note that $\zeta^{+}$and $\bar{\zeta}^{-}$have the same $\mathrm{U}(1)_{A} \mathrm{R}$-charge, and $e^{\sigma+i a}$ and $w$ are neutral under it.

The $\mathcal{N}=(2,2)$ supersymmetric background $\Sigma$ can be classified as follows according to the preserved supercharges given by the Killing spinors $\zeta^{\alpha}, \bar{\zeta}^{\alpha}$.

The first class of backgrounds preserves one supercharge of a given $\mathrm{U}(1)_{A}$ charge. Without loss of generality we can take the Killing spinors as nonzero $\left(\zeta^{+}, \bar{\zeta}^{-}\right)$and $\bar{\zeta}^{+}=$ 
$\zeta^{-}=0$. We find for every $\sigma$ and $a$

$$
\begin{aligned}
& \Sigma=\sigma+i a-2 i \theta^{2} \frac{\bar{\zeta}^{-}}{\zeta^{+}} \partial_{--}\left(\sigma+i a+\log \bar{\zeta}^{-}\right) \\
& \bar{\Sigma}=\sigma-i a+2 i \bar{\theta}^{2} \frac{\zeta^{+}}{\bar{\zeta}^{-}} \partial_{++}\left(\sigma-i a+\log \zeta^{+}\right)
\end{aligned}
$$

where $\zeta^{+}=\zeta^{+}\left(x^{++}\right)$and $\bar{\zeta}^{-}=\bar{\zeta}^{-}\left(x^{--}\right)$are arbitrary functions of $x^{++}$and $x^{--}$respectively. (In Euclidean space they are holomorphic and anti-holomorphic functions.) Recall that our analysis is local; global considerations restrict these functions.

The second class of backgrounds preserves two supercharges with opposite $\mathrm{U}(1)_{A} \mathrm{R}$ charge, denoted as $\left(\zeta_{1}^{+}, \bar{\zeta}_{1}^{-}\right)$and $\left(\bar{\zeta}_{2}^{+}, \zeta_{2}^{-}\right)$. Imposing invariance under $\left(\zeta_{1}^{+}, \bar{\zeta}_{1}^{-}\right)$we find again (D.2) with $\zeta \rightarrow \zeta_{1}$. Imposing invariance under $\left(\bar{\zeta}_{2}^{+}, \zeta_{2}^{-}\right)$we find another expression, which is related to (D.2) by $\zeta \rightarrow \bar{\zeta}_{2}, w \leftrightarrow \bar{w}, a \rightarrow-a$. Consistency of the two solutions constrains $\sigma$ and $a$

$$
\left(\zeta_{1}^{+} \bar{\zeta}_{2}^{+} \partial_{++}+\zeta_{2}^{-} \bar{\zeta}_{1}^{-} \partial_{--}\right)\left(\sigma+i a+\log \bar{\zeta}_{2}^{+}\left(x^{++}\right) \bar{\zeta}_{1}^{-}\left(x^{--}\right)\right)=0
$$

This means that $\sigma$ and $a$ are invariant under the vector $v \equiv \zeta_{1}^{+} \bar{\zeta}_{2}^{+} \partial_{++}+\zeta_{2}^{-} \bar{\zeta}_{1}^{-} \partial_{--}$(up to a superconformal transformation by $\left.\log \bar{\zeta}_{2}^{+}\left(x^{++}\right) \bar{\zeta}_{1}^{-}\left(x^{--}\right)\right)$.

There are two such cases depending on whether $v=0$ or $v \neq 0$. They lead to the topological twist and $\Omega$-deformation respectively.

For $v=0$ it suffices to assume that one component of $v$ vanishes. Without loss of generality take $\zeta_{1}^{+}=0$ with nonzero $\bar{\zeta}_{1}^{-}$. The solution (D.2) gives

$$
\Sigma=\theta^{2} w, \quad \bar{\Sigma}=2 \sigma,
$$

i.e. $a=i \sigma, \bar{w}=0$ with arbitrary $\sigma$ and $w$. This is the topological twist. The anti-topological twist corresponds to $a=-i \sigma$.

In the second case $v \neq 0$ and $\sigma$ and $a$ must have an isometry given by the vector $v$. Up to a conformal transformation we can take the Killing spinors to be $\left(\zeta_{1}^{+}, \bar{\zeta}_{1}^{-}\right)=$ $\left(\epsilon x^{++}, 1\right)$ and $\left(\bar{\zeta}_{2}^{+}, \zeta_{2}^{-}\right)=\left(1,-\epsilon x^{--}\right)$. Here $\epsilon$ is a constant describing the $\Omega$-deformation. The corresponding isometry vector is $v=\epsilon\left(x^{++} \partial_{++}-x^{--} \partial_{--}\right)$. Therefore, $\sigma$ and $a$ are arbitrary function of the invariant combination $x^{++} x^{--}$. The supersymmetry algebra satisfies $\delta_{1} \delta_{2}+\delta_{2} \delta_{1}=i \mathcal{L}_{v} \neq 0$. And the background is

$$
\begin{aligned}
& \Sigma=(\sigma+i a)-\frac{2 i}{\epsilon} \theta^{2} \frac{1}{x^{++}} \partial_{--}(\sigma+i a), \\
& \bar{\Sigma}=(\sigma-i a)+2 i \epsilon \bar{\theta}^{2} x^{++} \partial_{++}\left(\sigma-i a+\log x^{++}\right) .
\end{aligned}
$$

Two limiting cases are interesting. For $\epsilon \rightarrow 0$, the solution (D.5) becomes the topological background (D.4). For $a=i \sigma$ with nonzero $\epsilon$, equation (D.5) reproduces the two-dimensional $\Omega$-background in [44]

$$
\Sigma=0, \quad \bar{\Sigma}=2 \sigma+2 i \epsilon \bar{\theta}^{2} x^{--} \partial_{--}\left(2 \sigma+\log x^{--}\right) .
$$


The background (D.5) preserves maximally four supercharges, if and only if the spacetime metric is maximally symmetric and the $\mathrm{U}(1)_{A}$ gauge field has zero curvature $\partial_{++} \partial_{--} a=0$. For a sphere the maximally supersymmetric background is given by

$$
\begin{aligned}
& \Sigma=-\log \left(1+x^{2}\right)+\theta^{2} \frac{2 i / \epsilon}{1+x^{2}} \\
& \bar{\Sigma}=-\log \left(1+x^{2}\right)+\bar{\theta}^{2} \frac{2 i \epsilon}{1+x^{2}},
\end{aligned}
$$

where $x^{2} \equiv x^{++} x^{--}$. The sphere background (D.7) is not unitary since $\Sigma^{*} \neq \bar{\Sigma}$. The result agrees with the supersymmetric sphere background of [11, 12].

Similarly we can classify the $\mathcal{N}=(0,2)$ supersymmetric backgrounds. They are determined by imposing the vanishing right-moving supersymmetry variation on the chiral superfield $e^{\Sigma}=e^{\sigma+i a}$

$$
\begin{aligned}
\partial_{--} \zeta^{+}=\partial_{--} \bar{\zeta}^{+} & =0, \\
\partial_{++}\left(e^{\sigma+i a} \bar{\zeta}^{+}\right) & =0, \partial_{++}\left(e^{\sigma-i a} \zeta^{+}\right)=0 .
\end{aligned}
$$

There is only one class of $\mathcal{N}=(0,2)$ smooth supersymmetry backgrounds. They are referred to as (anti-) topological half-twist in [58].

Open Access. This article is distributed under the terms of the Creative Commons Attribution License (CC-BY 4.0), which permits any use, distribution and reproduction in any medium, provided the original author(s) and source are credited.

\section{References}

[1] A.B. Zamolodchikov, Irreversibility of the Flux of the Renormalization Group in a $2 D$ Field Theory, JETP Lett. 43 (1986) 730 [Pisma Zh. Eksp. Teor. Fiz. 43 (1986) 565] [INSPIRE].

[2] N. Seiberg, Observations on the Moduli Space of Superconformal Field Theories, Nucl. Phys. B 303 (1988) 286 [INSPIRE].

[3] D. Kutasov, Geometry on the Space of Conformal Field Theories and Contact Terms, Phys. Lett. B 220 (1989) 153 [inSPIRE].

[4] Y. Tachikawa, Five-dimensional supergravity dual of a-maximization, Nucl. Phys. B 733 (2006) 188 [hep-th/0507057] [INSPIRE].

[5] H. Osborn, Weyl consistency conditions and a local renormalization group equation for general renormalizable field theories, Nucl. Phys. B 363 (1991) 486 [INSPIRE].

[6] J. Wess and B. Zumino, Consequences of anomalous Ward identities, Phys. Lett. B 37 (1971) 95 [INSPIRE].

[7] J. Gomis, Z. Komargodski, H. Ooguri, N. Seiberg and Y. Wang, work in progress.

[8] H. Jockers, V. Kumar, J.M. Lapan, D.R. Morrison and M. Romo, Two-Sphere Partition Functions and Gromov-Witten Invariants, Commun. Math. Phys. 325 (2014) 1139 [arXiv: 1208.6244] [INSPIRE].

[9] J. Gomis and S. Lee, Exact Kähler Potential from Gauge Theory and Mirror Symmetry, JHEP 04 (2013) 019 [arXiv: 1210.6022] [InSPIRE]. 
[10] E. Gerchkovitz, J. Gomis and Z. Komargodski, Sphere Partition Functions and the Zamolodchikov Metric, JHEP 11 (2014) 001 [arXiv:1405.7271] [INSPIRE].

[11] F. Benini and S. Cremonesi, Partition Functions of $\mathcal{N}=(2,2)$ Gauge Theories on $S^{2}$ and Vortices, Commun. Math. Phys. 334 (2015) 1483 [arXiv:1206.2356] [InSPIRE].

[12] N. Doroud, J. Gomis, B. Le Floch and S. Lee, Exact Results in D $=2$ Supersymmetric Gauge Theories, JHEP 05 (2013) 093 [arXiv:1206.2606] [inSPIRE].

[13] N. Doroud and J. Gomis, Gauge theory dynamics and Kähler potential for Calabi-Yau complex moduli, JHEP 12 (2013) 99 [arXiv:1309.2305] [INSPIRE].

[14] J. Gomis and N. Ishtiaque, Kähler potential and ambiguities in $4 d \mathcal{N}=2$ SCFTs, JHEP 04 (2015) 169 [arXiv:1409.5325] [INSPIRE].

[15] M. Baggio, V. Niarchos and K. Papadodimas, $t t^{*}$ equations, localization and exact chiral rings in $4 d \mathcal{N}=2$ SCFTs, JHEP 02 (2015) 122 [arXiv:1409.4212] [INSPIRE].

[16] M. Baggio, V. Niarchos and K. Papadodimas, Exact correlation functions in $\mathrm{SU}(2) \mathcal{N}=2$ superconformal QCD, Phys. Rev. Lett. 113 (2014) 251601 [arXiv:1409.4217] [INSPIRE].

[17] M. Baggio, V. Niarchos and K. Papadodimas, On exact correlation functions in $\mathrm{SU}(N)$ $\mathcal{N}=2$ superconformal QCD, JHEP 11 (2015) 198 [arXiv:1508.03077] [INSPIRE].

[18] M. Buican and T. Nishinaka, Compact Conformal Manifolds, JHEP 01 (2015) 112 [arXiv: 1410.3006] [INSPIRE].

[19] C. Closset, T.T. Dumitrescu, G. Festuccia, Z. Komargodski and N. Seiberg, Contact Terms, Unitarity and F-Maximization in Three-Dimensional Superconformal Theories, JHEP 10 (2012) 053 [arXiv: 1205.4142] [INSPIRE].

[20] C. Closset, T.T. Dumitrescu, G. Festuccia, Z. Komargodski and N. Seiberg, Comments on Chern-Simons Contact Terms in Three Dimensions, JHEP 09 (2012) 091 [arXiv: 1206.5218] [INSPIRE].

[21] R. Auzzi and B. Keren-Zur, Superspace formulation of the local RG equation, JHEP 05 (2015) 150 [arXiv: 1502.05962] [INSPIRE].

[22] Y. Nakayama, Consistency of local renormalization group in D $=3$, Nucl. Phys. B $\mathbf{8 7 9}$ (2014) 37 [arXiv: 1307.8048] [INSPIRE].

[23] D. Friedan and A. Konechny, Curvature formula for the space of 2-D conformal field theories, JHEP 09 (2012) 113 [arXiv:1206.1749] [INSPIRE].

[24] L. Bonora, P. Pasti and M. Bregola, Weyl Cocycles, Class. Quant. Grav. 3 (1986) 635 [INSPIRE].

[25] S. Deser and A. Schwimmer, Geometric classification of conformal anomalies in arbitrary dimensions, Phys. Lett. B 309 (1993) 279 [hep-th/9302047] [INSPIRE].

[26] I. Jack and H. Osborn, Analogs for the c Theorem for Four-dimensional Renormalizable Field Theories, Nucl. Phys. B 343 (1990) 647 [INSPIRE].

[27] B. Grinstein, A. Stergiou and D. Stone, Consequences of Weyl Consistency Conditions, JHEP 11 (2013) 195 [arXiv: 1308.1096] [InSPIRE].

[28] I. Jack and H. Osborn, Constraints on RG Flow for Four Dimensional Quantum Field Theories, Nucl. Phys. B 883 (2014) 425 [arXiv:1312.0428] [InSPIRE]. 
[29] F. Baume, B. Keren-Zur, R. Rattazzi and L. Vitale, The local Callan-Symanzik equation: structure and applications, JHEP 08 (2014) 152 [arXiv:1401.5983] [INSPIRE].

[30] B. Grinstein, D. Stone, A. Stergiou and M. Zhong, Challenge to the a Theorem in Six Dimensions, Phys. Rev. Lett. 113 (2014) 231602 [arXiv:1406.3626] [INSPIRE].

[31] H. Osborn and A. Stergiou, Structures on the Conformal Manifold in Six Dimensional Theories, JHEP 04 (2015) 157 [arXiv:1501.01308] [INSPIRE].

[32] Y. Nakayama, Scale invariance vs conformal invariance, Phys. Rept. 569 (2015) 1 [arXiv: 1302.0884] [INSPIRE].

[33] E.S. Fradkin and A.A. Tseytlin, Asymptotic Freedom In Extended Conformal Supergravities, Phys. Lett. B 110 (1982) 117 [inSPIRE].

[34] E.S. Fradkin and A.A. Tseytlin, One Loop $\beta$-function in Conformal Supergravities, Nucl. Phys. B 203 (1982) 157 [INSPIRE].

[35] S.M. Paneitz, A quartic conformally covariant differential operator for arbitrary peudo-Riemannian manifolds, SIGMA 4 (2008) 036 [arXiv:0803.4331].

[36] R.J. Riegert, A Nonlocal Action for the Trace Anomaly, Phys. Lett. B 134 (1984) 56 [INSPIRE].

[37] Y. Nakayama, Local renormalization group functions from quantum renormalization group and holographic bulk locality, JHEP 06 (2015) 092 [arXiv: 1502.07049] [INSPIRE].

[38] N. Seiberg, Naturalness versus supersymmetric nonrenormalization theorems, Phys. Lett. B 318 (1993) 469 [hep-ph/9309335] [INSPIRE].

[39] G. Festuccia and N. Seiberg, Rigid Supersymmetric Theories in Curved Superspace, JHEP 06 (2011) 114 [arXiv: 1105.0689] [INSPIRE].

[40] P.S. Howe and G. Papadopoulos, $N=2, D=2$ Supergeometry, Class. Quant. Grav. 4 (1987) 11 [INSPIRE].

[41] M.T. Grisaru and M.E. Wehlau, Prepotentials for (2,2) supergravity, Int. J. Mod. Phys. A 10 (1995) 753 [hep-th/9409043] [INSPIRE].

[42] M.T. Grisaru and M.E. Wehlau, Superspace measures, invariant actions and component projection formulae for (2,2) supergravity, Nucl. Phys. B 457 (1995) 219 [hep-th/9508139] [INSPIRE].

[43] S.J. Gates, Jr., M.T. Grisaru and M.E. Wehlau, A study of general 2-D, $N=2$ matter coupled to supergravity in superspace, Nucl. Phys. B 460 (1996) 579 [hep-th/9509021] [INSPIRE].

[44] C. Closset and S. Cremonesi, Comments on $\mathcal{N}=(2,2)$ supersymmetry on two-manifolds, JHEP 07 (2014) 075 [arXiv: 1404.2636] [INSPIRE].

[45] E. Witten and J. Bagger, Quantization of Newton's Constant in Certain Supergravity Theories, Phys. Lett. B 115 (1982) 202 [INSPIRE].

[46] Z. Komargodski and N. Seiberg, Comments on the Fayet-Iliopoulos Term in Field Theory and Supergravity, JHEP 06 (2009) 007 [arXiv:0904.1159] [INSPIRE].

[47] Z. Komargodski and N. Seiberg, Comments on Supercurrent Multiplets, Supersymmetric Field Theories and Supergravity, JHEP 07 (2010) 017 [arXiv: 1002.2228] [InSPIRE]. 
[48] N. Seiberg, Modifying the Sum Over Topological Sectors and Constraints on Supergravity, JHEP 07 (2010) 070 [arXiv: 1005.0002] [INSPIRE].

[49] T. Banks and N. Seiberg, Symmetries and Strings in Field Theory and Gravity, Phys. Rev. D 83 (2011) 084019 [arXiv: 1011.5120] [InSPIRE].

[50] N.A. Nekrasov, Lectures on curved beta-gamma systems, pure spinors and anomalies, hep-th/0511008 [INSPIRE].

[51] E. Witten, Topological $\sigma$-models, Commun. Math. Phys. 118 (1988) 411 [INSPIRE].

[52] E. Witten, Mirror manifolds and topological field theory, In Mirror symmetry I, S.T. Yau ed., pg. 121-160, hep-th/9112056 [INSPIRE].

[53] M. Bershadsky, S. Cecotti, H. Ooguri and C. Vafa, Kodaira-Spencer theory of gravity and exact results for quantum string amplitudes, Commun. Math. Phys. 165 (1994) 311 [hep-th/9309140] [INSPIRE].

[54] F. Benini and A. Zaffaroni, A topologically twisted index for three-dimensional supersymmetric theories, JHEP 07 (2015) 127 [arXiv: 1504.03698] [INSPIRE].

[55] C. Closset, S. Cremonesi and D.S. Park, The equivariant A-twist and gauged linear $\sigma$-models on the two-sphere, JHEP 06 (2015) 076 [arXiv:1504.06308] [INSPIRE].

[56] C.P. Bachas, I. Brunner, M.R. Douglas and L. Rastelli, Calabi's diastasis as interface entropy, Phys. Rev. D 90 (2014) 045004 [arXiv: 1311.2202] [INSPIRE].

[57] M. Bertolini, I.V. Melnikov and M.R. Plesser, Accidents in (0,2) Landau-Ginzburg theories, JHEP 12 (2014) 157 [arXiv:1405.4266] [INSPIRE].

[58] E. Witten, Phases of $N=2$ theories in two-dimensions, Nucl. Phys. B 403 (1993) 159 [hep-th/9301042] [INSPIRE].

[59] V. Periwal and A. Strominger, Kähler Geometry of the Space of $N=2$ Superconformal Field Theories, Phys. Lett. B 235 (1990) 261 [InSPIRE].

[60] T.T. Dumitrescu and N. Seiberg, Supercurrents and Brane Currents in Diverse Dimensions, JHEP 07 (2011) 095 [arXiv: 1106.0031] [INSPIRE].

[61] S. Groot Nibbelink and L. Horstmeyer, Super Weyl invariance: BPS equations from heterotic worldsheets, JHEP 07 (2012) 054 [arXiv: 1203.6827] [INSPIRE].

[62] B. de Wit, J.W. van Holten and A. Van Proeyen, Structure of $N=2$ Supergravity, Nucl. Phys. B 184 (1981) 77 [Erratum ibid. B 222 (1983) 516] [INSPIRE].

[63] S.M. Kuzenko, Super-Weyl anomalies in $N=2$ supergravity and (non)local effective actions, JHEP 10 (2013) 151 [arXiv:1307.7586] [INSPIRE].

[64] B. de Wit, S. Katmadas and M. van Zalk, New supersymmetric higher-derivative couplings: Full $N=2$ superspace does not count!, JHEP 01 (2011) 007 [arXiv:1010.2150] [INSPIRE].

[65] D. Butter, B. de Wit, S.M. Kuzenko and I. Lodato, New higher-derivative invariants in $N=2$ supergravity and the Gauss-Bonnet term, JHEP 12 (2013) 062 [arXiv:1307.6546] [INSPIRE].

[66] J. Louis, H. Triendl and M. Zagermann, $\mathcal{N}=4$ supersymmetric $A d S_{5}$ vacua and their moduli spaces, JHEP 10 (2015) 083 [arXiv:1507.01623] [INSPIRE]. 